\title{
Asymptotic Regularity of Solutions of Hartree-Fock Equations with Coulomb Potential
}

\author{
Heinz-Jürgen Flad and Reinhold Schneider \\ Institut für Informatik, Christian-Albrechts-Universität Kiel, Christian-Albrechts-Platz 4, D-24098 Kiel \\ Bert-Wolfgang Schulze \\ Institut für Mathematik, Universität Potsdam, Am Neuen Palais 10, D-14469 Potsdam
}

May 23, 2007

\begin{abstract}
We study the asymptotic regularity of solutions of Hartree-Fock equations for Coulomb systems. In order to deal with singular Coulomb potentials, Fock operators are discussed within the calculus of pseudo-differential operators on conical manifolds. First, the non-self-consistent-field case is considered which means that the functions that enter into the nonlinear terms are not the eigenfunctions of the Fock operator itself. We introduce asymptotic regularity conditions on the functions that build up the Fock operator which guarantee ellipticity for the local part of the Fock operator on the open stretched cone $\mathbb{R}_{+} \times S^{2}$. This proves existence of a parametrix with a corresponding smoothing remainder from which it follows, via a bootstrap argument, that the eigenfunctions of the Fock operator again satisfy asymptotic regularity conditions. Using a fixed-point approach based on Cancès and Le Bris analysis of the level-shifting algorithm, we show via another bootstrap argument, that the corresponding self-consistent-field solutions of the Hartree-Fock equation have the same type of asymptotic regularity.
\end{abstract}

Keywords: Hartree-Fock equation, conical singularities, asymptotics of eigenfunctions

Mathematics Subject Classification: 35Q40, 35C20, 35J10

\section{Hartree-Fock equation on the open stretched cone}

\subsection{Outline of the Hartree-Fock model}

The nonrelativistic Schrödinger equation within the Born-Oppenheimer approximation provides a firm basis for electronic structure calculations in quantum chemistry. We are focusing on solutions of the stationary Schrödinger equation

$$
H \Psi\left(\underline{x}_{1}, \underline{x}_{2}, \ldots, \underline{x}_{N}\right)=E \Psi\left(\underline{x}_{1}, \underline{x}_{2}, \ldots, \underline{x}_{N}\right),
$$

where the Hamiltonian for an $N$ electron system

$$
H=\sum_{i=1}^{N}\left(-\frac{1}{2} \Delta_{i}-\sum_{k=1}^{K} \frac{Z_{k}}{\left|x_{i}-R_{k}\right|}\right)+\sum_{i<j} \frac{1}{\left|x_{i}-x_{j}\right|}
$$

includes Coulomb interactions between the electrons as well as external Coulomb potentials due to the presence of nuclei with charge $Z_{k}$ at $R_{k} \in \mathbb{R}^{3}$. Atomic units have been used throughout the present work. The Hamiltonian does not contain spin-dependent interactions, like spin-orbit coupling, therefore there is no immediate need to introduce spin degrees of freedom. Nevertheless it 
is convenient to express the wavefunction $\Psi$ in terms of combined spatial and spin coordinates $\underline{x}_{i}:=$ $\left(x_{i}, \chi\right)$ for $i=1, \ldots, N$, with $x_{i} \in \mathbb{R}^{3}, \chi \in\{1 / 2,-1 / 2\}$ in order to achieve a simple representation of Fermi statistics. The latter can be formulated via Pauli's principle which states that $\Psi$ must be an antisymmetric function with respect to the variables $\underline{x}_{i}$ for $i=1, \ldots, N$.

$A b$ initio quantum chemistry deals with approximate models of the many-electron Schrödinger equation (1.1) without referring to external experimental or computational data. A prime example for such kind of approach is the Hartree-Fock (HF) model which can be considered as a constraint variational formulation of the original many-electron problem. Within the HF model, the $N$-electron wavefunction is approximated by a so-called Slater determinant

$$
\Phi=1 / \sqrt{N !} \operatorname{det}\left[\phi_{i}\left(\underline{x}_{j}\right)\right]_{i, j=1, \ldots, N} \text { with }\left\langle\phi_{i} \mid \phi_{j}\right\rangle=\delta_{i, j},
$$

which represents an ansatz to construct a many-electron wavefunction $\Phi$, consistent with Pauli's principle, from a set of single-electron wavefunctions $\left\{\phi_{i}\right\}_{i=1, \ldots, N}$. Here and in the following scalar products $\langle\mid\rangle$ refer to the standard Hilbert space in quantum theory $L^{2}$. By definition, the HF energy is given by

$$
E_{\mathrm{HF}}:=\inf \left\{E[\Phi]:=\langle\Phi \mid H \Phi\rangle: \Phi \text { Slater determinant, } \phi_{i} \in H^{1}\left(\mathbb{R}^{3} \times\{ \pm 1 / 2\}\right)\right\} .
$$

From the work of Lieb, Simon and Lions $[8,9]$ it is known that a minimizer $E_{\mathrm{HF}}=E\left[\Phi_{\mathrm{HF}}\right]$ exists. Furthermore they have shown that the functions $\phi_{i}$ are globally Lipschitz, actually smooth except at the nuclei, and decay exponentially at infinity. The variational formulation of the HF method leads to the nonlinear HF equation

$$
\mathfrak{h} \phi_{i}=\varepsilon_{i} \phi_{i}, \text { with } \phi_{i} \in H^{1}\left(\mathbb{R}^{3}\right),
$$

which corresponds to an effective one-particle Schrödinger equation with Hamiltonian

$$
\begin{gathered}
\mathfrak{h}=-\frac{1}{2} \Delta-\sum_{k=1}^{K} \frac{Z_{k}}{\left|x-R_{k}\right|}+V_{H}+\mathcal{U}, \\
V_{H}(x)=2 \sum_{i=1}^{N / 2} \int \frac{\left|\phi_{i}(y)\right|^{2}}{|x-y|} d y \quad \text { (Hartree potential), } \\
\mathcal{U} u(x)=-\sum_{i=1}^{N / 2} \int \frac{\phi_{i}(x) \phi_{i}(y) u(y)}{|x-y|} d y \quad \text { (exchange operator). }
\end{gathered}
$$

In the following the Hamiltonian (1.4) is denoted as Fock operator. We restrict our discussion to the closed shell HF equation for an even number $N$ of electrons, where spin degrees of freedom are not taken into account explicitly. Each spatial eigenfunction $\phi_{i}$ of the Fock operator belongs to two eigenstates because of the electron spin. Therefore, the sums in the Hartree potential (1.5) and exchange operator (1.6) run over the $N / 2$ eigenfunctions with lowest eigenvalue $\varepsilon_{i}$.

With the emergence of powerful computers, the HF model became a workhorse of computational chemists and countless applications have been reported in the literature. Furthermore the HF model provides a convenient starting point for more sophisticated many-particle theories. We refer to the monograph [6] for a state of the art discussion of these topics. Despite of its outstanding significance for computational chemistry, there is still a lack of rigorous results concerning the approximation of HF eigenfunctions. An attempt in this direction has been presented in Ref. [4] for tensor product wavelets within the framework of best $N$-term approximation theory. Due to a lack of knowledge concerning the asymptotic behaviour, it was however necessary to assume an asymptotic smoothness property

$$
\left|\partial_{x}^{\beta} \phi_{i}(x)\right| \lesssim\left|x-R_{k}\right|^{1-|\beta|} \text { for } \Omega_{k} \ni x \neq R_{k} \text { and }|\beta| \geq 1
$$


for HF eigenfunctions in bounded neighbourhoods $\Omega_{k} \subset \mathbb{R}^{3}$ of the nuclei. Here and in the following we have used the standard short-hand notation for mixed partial derivatives

$$
\partial^{\beta}:=\frac{\partial^{\beta_{1}}}{\partial x_{1}^{\beta_{1}}} \frac{\partial^{\beta_{2}}}{\partial x_{2}^{\beta_{2}}} \frac{\partial^{\beta_{3}}}{\partial x_{3}^{\beta_{3}}}
$$

with absolute value of the multi-index $|\beta|:=\beta_{1}+\beta_{2}+\beta_{3}$. Furthermore, $a \lesssim b$ means that $a$ is uniformly bounded by some constant multiple of $b$. A verification of assumption (1.7) within the self-consistent solution scheme discussed below, cf. Proposition 1, is a simple byproduct of our asymptotic analysis.

In order to study the asymptotic behaviour of eigenfunctions, we formally consider the underlying $\mathbb{R}^{3}$ as a manifold with conical singularities at the locations of the nuclei. This can be realized by introducing charts with polar coordinates in possibly unbounded neighbourhoods of the nuclei. We refer to Ref. [3] for further details concerning manifold with conical singularities. Our basic motivation for this seemingly artificial construction are the singular parts of the Fock operator which can be handled within this setting in a natural manner as it is demonstrated below. Since such kind of construction can be always carried out for each nucleus separately, we restrict in the following our discussion to a single nucleus. To be more specific we consider the HF equation on the open stretched cone $X^{\wedge}:=\mathbb{R}_{+} \times X$ with $X=S^{2}$. The quotient $X^{\triangle}:=\left(\overline{\mathbb{R}}_{+} \times S^{2}\right) /\left(0 \times S^{2}\right)$ corresponds to a manifold with conical point at the orgin which can be identified with $\mathbb{R}^{3}$. For the convenience of the reader and in order to keep the paper reasonably self-contained, we provide some background material on the calculus of pseudo-differential operators on conical manifolds in Section 3 .

\subsection{Weighted Sobolev spaces with asymptotics on $X^{\wedge}$}

For further reference, we recall some basic definitions of weighted Sobolev spaces on $X^{\wedge}$, and refer e.g. to Ref. [3] for a general exposition and to the monograph [10] for a comprehensive treatment of the subject. It should be mentioned that for the convenience of the reader, we follow with our notation closely Ref. [3]. The open stretched cone $X^{\wedge}$ can be considered as a half space of the infinite cylinder on which we introduce the standard Sobolev spaces

$$
H_{\text {cone }}^{s}\left(X^{\wedge}\right):=\left\{\left.u \in H_{\text {loc }}^{s}\left(\mathbb{R} \times S^{2}\right)\right|_{\mathbb{R}_{+} \times S^{2}}:(1-\omega) u \in H^{s}\left(\mathbb{R}^{3}\right)\right\},
$$

for any cut-off function $\omega$, i.e. $\omega \equiv 1$ near $0, \omega \equiv 0$ outside some neighbourhood of 0 . Furthermore, we introduce the weighted Sobolev spaces with $s \in \mathbb{Z}_{+}$and $\gamma \in \mathbb{R}$

$$
\mathcal{H}^{s, \gamma}\left(X^{\wedge}\right):=\left\{u \in \mathcal{D}^{\prime}\left(X^{\wedge}\right):|x|^{|\alpha|} \partial^{\alpha} u \in|x|^{\gamma} L^{2}\left(\mathbb{R}^{3}\right) \text { for all }|\alpha| \leq s\right\} .
$$

According to Remark 2.1.13 of Ref. [10], the definition (1.9) is equivalent to the original definition using the Mellin transform. It is convenient to introduce the corresponding norm

$$
\|u\|_{\mathcal{H}^{s, \gamma}\left(X^{\wedge}\right)}:=\sum_{|\alpha| \leq s}\left\||x|^{-\gamma+|\alpha|} \partial^{\alpha} u\right\|_{L^{2}\left(\mathbb{R}^{3}\right)} .
$$

Neither the Sobolev space (1.8) nor (1.9) is really appropriate for the infinite open stretched cone $X^{\wedge}$. Instead it is better to consider the combination

$$
\mathcal{K}^{s, \gamma}\left(X^{\wedge}\right):=\omega \mathcal{H}^{s, \gamma}\left(X^{\wedge}\right)+(1-\omega) H_{\text {cone }}^{s}\left(X^{\wedge}\right),
$$

which provides the appropriate asymptotic behaviour in the limits $|x| \rightarrow 0$ and $|x| \rightarrow \infty$, respectively. 
Next we consider subspaces of $\mathcal{K}^{s, \gamma}\left(X^{\wedge}\right)$ of certain asymptotic type in the vicinity of the tip of the cone. For this it is convenient to introduce polar coordinates $(r, \phi, \theta)$. These subspaces consist of functions with asymptotic expansions

$$
u(r, \phi, \theta) \sim \sum_{j} \sum_{k=0}^{m_{j}} c_{j k}(\phi, \theta) r^{-p_{j}} \ln ^{k} r
$$

where $c_{j k}$ belong to finite dimensional subspaces $L_{j} \subset C^{\infty}\left(S^{2}\right)$ and $p_{j} \in \mathbb{C}$ are taken from a strip of the complex plane, i.e.

$$
p_{j} \in\left\{z: \frac{3}{2}-\gamma+\vartheta<\Re z<\frac{3}{2}-\gamma\right\}
$$

The width and location of a strip are determined by it's weight data $(\gamma, \Theta)$ with $\Theta=(\vartheta, 0]$ and $-\infty \leq \vartheta<0$, where each substrip of finite width contains only a finite number of $p_{j}$. An asymptotic expansion (1.11) is completely characterized by the asymptotic type $P:=\left\{\left(p_{j}, m_{j}, L_{j}\right)\right\}_{j \in \mathbb{Z}_{+}}$. Together, weight data $(\gamma, \Theta)$ and asymptotic type $P$ define weighted Sobolev spaces with asymptotics

$$
\mathcal{K}_{P}^{s, \gamma}\left(X^{\wedge}\right):=\left\{u \in \mathcal{K}^{s, \gamma}\left(X^{\wedge}\right): u-\omega \sum_{j} \sum_{k=0}^{m_{j}} c_{j k}(\phi, \theta) r^{-p_{j}} \ln ^{k} r \in \mathcal{K}_{\Theta}^{s, \gamma}\left(X^{\wedge}\right)\right\},
$$

where

$$
\mathcal{K}_{\Theta}^{s, \gamma}\left(X^{\wedge}\right):=\bigcap_{\epsilon>0} \mathcal{K}^{s, \gamma-\vartheta-\epsilon}\left(X^{\wedge}\right) .
$$

The sum in (1.12) is taken over those $j$ such that $\Re p_{j}>\frac{3}{2}-\gamma+\vartheta$. Alternatively these spaces can be considered as a direct sum of the spaces

$$
\mathcal{K}_{P}^{s, \gamma}\left(X^{\wedge}\right)=\mathcal{E}_{P}^{\gamma}+\mathcal{K}_{\Theta}^{s, \gamma}\left(X^{\wedge}\right) \text { with } \mathcal{E}_{P}^{\gamma}:=\left\{\omega \sum_{j} \sum_{k=0}^{m_{j}} c_{j k}(\phi, \theta) r^{-p_{j}} \ln ^{k} r\right\} .
$$

intersecting in $\{0\}$. Furthermore, we employ the asymptotic spaces

$$
\mathcal{S}_{P}^{\gamma}\left(X^{\wedge}\right):=\left\{u \in \mathcal{K}_{P}^{\infty, \gamma}\left(X^{\wedge}\right):\left.(1-\omega) u \in \mathcal{S}\left(\mathbb{R}, C^{\infty}\left(S^{2}\right)\right)\right|_{\mathbb{R}_{+} \times S^{2}}\right\},
$$

with Schwartz class type of behaviour for exit $r \rightarrow \infty$. The spaces (1.12) and (1.14) are Fréchet spaces equipped with natural quasi-norms according to the decomposition (1.13), we refer to Refs. $[3,10]$ for further datails.

\section{Basic definitions and main theorems}

The basic statement of the paper concerns the iterative solution of the HF equation (1.3) within a function class of a certain asymptotic type $P$.

Definition 1. A function $u$ is asymptotically well behaved if $u \in \mathcal{S}_{P}^{\gamma}\left(X^{\wedge}\right)$ for $\gamma<\frac{3}{2}$, asymptotic type $P=\left\{\left(-j, 0, L_{j}\right)\right\}_{j \in \mathbb{Z}_{+}}$and associated weight data $(\gamma, \Theta)$ with $\Theta=(-\infty, 0]$. The finite dimensional spaces $L_{j} \subset C^{\infty}\left(S^{2}\right)$ are given by $L_{j}=\operatorname{span}\left\{Y_{l, m}: l \leq j\right\}$, where $Y_{l, m}$ denotes spherical harmonics on $S^{2}$.

Proposition 1. An asymptotically well behaved function u belongs to $C^{\infty}\left(\overline{\mathbb{R}}_{+} \times S^{2}\right)$ and possesses the asymptotic smoothness property (1.7). 
Proof. The function $u$ can be written as

$$
u(r, \phi, \theta)=\underbrace{\sum_{j=0}^{k} c_{j}(\phi, \theta) r^{j} \omega(r)}_{\in C^{\infty}\left(\overline{\mathbb{R}}_{+} \times S^{2}\right)}+\Phi_{k+1}(r, \phi, \theta),
$$

where the remainder $\Phi_{k+1}$ belongs to $\mathcal{K}^{s, \delta}\left(X^{\wedge}\right)$ for $\delta<5 / 2+k$ and $s \in \mathbb{R}$. For $k$ sufficiently large, $\omega \Phi_{k+1} \in \mathcal{H}^{s, s}\left(\mathbb{R}_{+} \times S^{2}\right)$ with $s \in \mathbb{Z}_{+}$, which according to (1.9) is equivalent to

$$
\sum_{|\alpha| \leq s} \int_{\mathbb{R}^{3}}|x|^{2|\alpha|-2 s}\left|\partial^{\alpha}\left(\omega \Phi_{k+1}\right)\right|^{2} d x<\infty .
$$

This estimate together with (1.10) proves that $\Phi_{k+1}$ belongs to $H^{s}\left(\mathbb{R}^{3}\right)$. According to Sobolev's lemma, cf. Ref. [5], we have $H^{s}\left(\mathbb{R}^{3}\right) \hookrightarrow C_{B}^{m}\left(\mathbb{R}^{3}\right)$ for $m<s-2$, where $C_{B}^{m} \subset C^{m}$ denotes the space of bounded mixed derivatives. Therefore, $\Phi_{k+1}$ belongs to $C_{B}^{m}\left(\mathbb{R}^{3}\right)$ for $k>m-1 / 2$. The asymptotic smoothness property (1.7) is an obvious consequence.

Remark 1. The product uv of two asymptotically well behaved function $u, v$ is again asymptotically well behaved.

Various iteration schemes for the HF equation have been reported in the literature. We just want to mention the Roothaan, DIIS and level-shifting algorithms discussed in Ref. [1]. For the continous HF equation (1.3) only the level-shifting algorithm has been proven to converge to a solution [2]. The problem under which conditions such a solution actually corresponds to the global minimum of the original variational formulation (1.2) is still open. It can be only taken for granted that the solution either represents a possibly local minimum or at least a saddle point of the variational problem. The level-shifting algorithm follows the iterative scheme

$$
\cdots \longrightarrow\left\{\phi_{i}^{(n)}\right\}_{i=1, \ldots, N / 2} \longrightarrow \mathfrak{h}_{\text {shift }}^{(n)}:=\mathfrak{h}^{(n)}-b \mathcal{P}^{(n)} \longrightarrow\left\{\phi_{i}^{(n+1)}\right\}_{i=1, \ldots, N / 2} \longrightarrow \cdots
$$

where in each step, the $N / 2$ lowest eigenfunctions $\phi_{i}^{(n)}$ of a shifted Fock operator $\mathfrak{h}_{\text {shift }}^{(n-1)}$ are selected according to the aufbau principle in order to construct a new shifted Fock operator $\mathfrak{h}_{\text {shift }}^{(n)}$. Here the operator $\mathcal{P}^{(n)}$ denotes an orthogonal projection on the space spanned by the eigenfunctions $\left\{\phi_{i}^{(n)}\right\}_{i=1, \ldots, N / 2}$. The convergence of this algorithm (up to extractions), for a sufficiently large parameter $b$, to a "self-consistent-field" solution of the HF equation (1.3) has been proven by Cancès and Le Bris in Ref. [2]. In Theorem 11 of their paper, the convergence of the reduced one-particle density matrix, as a Hilbert-Schmidt operator on $H^{1}\left(\mathbb{R}^{3}\right)$, has been demonstrated in the corresponding operator norm. For simplicity, we assume in the following the absence of "accidental" degeneracies within the $N / 2$ lowest eigenvalues of the Fock operator, which means that in the case of a degenerate eigenvalue, the corresponding eigenfunctions can be uniquely characterized by symmetry. In this case it is possible to assign a unique set of eigenfunctions to the reduced one-particle density matrix. Otherwise it might happen that different subsequences converge to different sets of eigenfunctions, cf. the proof of Theorem 7 in Ref. [2]. These sets, however, are equivalent up to unitary transformations and give rise to the same Slater determinant.

The first theorem concerns the properties of the eigenfunctions $\phi_{i}^{(n)}$ in the intermediate steps of the level-shifting algorithm.

Theorem 1. The eigenfunctions $\left\{\phi_{i}^{(n+1)}\right\}_{i=1, \ldots, N / 2}$ of a shifted Fock operator $\mathfrak{h}_{\text {shift }}^{(n)}$ constructed from a set $\left\{\phi_{i}^{(n)}\right\}_{i=1, \ldots, N / 2}$ of asymptotically well behaved functions, are again asymptotically well behaved. 
Our central theorem is a consequence of Theorem 1 and the convergence property of the levelshifting algorithm.

Theorem 2. The self-consistent-field solutions of the HF equation (1.3), obtained via the levelshifting algorithm, are asymptotically well behaved provided that the initial guess $\left\{\phi_{i}^{(0)}\right\}_{i=1, \ldots, N / 2}$ possesses this property.

Such an initial guess can be obtained e.g. from the linear part of the Fock operator discussed in the next section.

\subsection{Ellipticity of local Fock operator on an infinite cone}

In order to prove Theorem 1, we have to show that the local part of the Fock operator constructed from a set $\left\{\phi_{i}^{(n)}\right\}_{i=1, \ldots, N / 2}$ of asymptotically well behaved functions belongs to the operator class $C^{2}\left(X^{\wedge}, g\right)$ for the weight data $\underline{g}=(\gamma, \gamma-2, \Theta), \gamma<3 / 2$ and $\Theta=(-\infty, 0]$, cf. Def. 1, p. 258 of Ref. [3], and is elliptic in the sense of Def. 6, p. 259 of Ref. [3]. We refer to Section 3.3 for a brief discussion of this operator calculus.

First we consider the linear part of the Fock operator for a nucleus located at the origin

$$
\mathfrak{h}_{\operatorname{lin}}=-\frac{1}{2} \Delta-\frac{Z}{|x|}+V_{\text {ext }}
$$

where $V_{\text {ext }}$ corresponds to an external potential which belongs to $C^{\infty}\left(\mathbb{R}^{3}\right)$ and satisfies asymptotic estimates of the form $\left|\partial^{\alpha} V_{\text {ext }}\right| \lesssim(1+|x|)^{-1-|\alpha|}$. On an open stretched cone $X^{\wedge}$ this operator can be expressed in polar coordinates $x \rightarrow(r, \theta, \phi)$ as a Fuchs type differential operator (cf. Ref. [3], p. 144f)

$$
\mathfrak{h}_{\text {lin }}=\frac{1}{r^{2}}\left[-\frac{1}{2}\left(-r \frac{\partial}{\partial r}\right)^{2}+\frac{1}{2}\left(-r \frac{\partial}{\partial r}\right)+\frac{1}{2} \mathfrak{l}^{2}-Z r+r^{2} V_{\text {ext }}\right],
$$

where $\mathfrak{l}^{2}$ corresponds to the total angular momentum operator on $S^{2}$, cf. Ref. [12].

Proposition 2. The linear part of the Fock operator $\mathfrak{h}_{\text {lin }}$ belongs to $C^{2}\left(X^{\wedge}, \underline{g}\right)$ for weight data $\underline{g}=(\gamma, \gamma-2, \Theta)$ with $\gamma \in \mathbb{R}$ and $\Theta=(-\infty, 0]$. Furthermore, the shifted operator $\mathfrak{h}_{\text {lin }}-\varepsilon$ for $\varepsilon<0$ is elliptic in the sense of Def. 6, p. 259 of Ref. [3] for $3 / 2-\gamma \in \mathbb{R} \backslash \mathbb{Z}$. There exists a parametrix $\mathcal{P}_{\text {lin }} \in C^{-2}\left(X^{\wedge}, \underline{h}\right)$ for $\underline{h}=(\gamma-2, \gamma, \Theta)$ and a corresponding Green operator $\mathcal{G}_{\text {lin }} \in C_{G}\left(X^{\wedge}, \underline{m}\right)$ for $\underline{m}=(\gamma, \gamma, \Theta)$ with

$$
\mathcal{P}_{\text {lin }}\left(\mathfrak{h}_{\text {lin }}-\varepsilon\right)=I+\mathcal{G}_{\text {lin }}
$$

which provide continous maps

$$
\mathcal{P}_{\text {lin }}: \mathcal{K}_{P}^{s, \gamma}\left(X^{\wedge}\right) \longrightarrow \mathcal{K}_{Q}^{s+2, \gamma+2}\left(X^{\wedge}\right) \text { and } \mathcal{G}_{\text {lin }}: \mathcal{K}^{s, \gamma}\left(X^{\wedge}\right) \longrightarrow \mathcal{S}_{Q}^{\gamma}\left(X^{\wedge}\right)
$$

Proof. Since $\mathfrak{h}_{\text {lin }}$ is a second order differential operator of Fuchs type, the first property follows immediately.

In order to prove ellipticity we have to check three different ellipticity conditions, cf. Section 3.2, which have to be fulfilled by various symbols defined for the operator class $C^{2}\left(X^{\wedge}, \underline{g}\right)$.

(i) The homogeneous principal symbol is given by

$$
\sigma_{\psi}^{2}\left(\mathfrak{h}_{\text {lin }}-\varepsilon\right)=\frac{1}{2 r^{2}}\left[(r \tau)^{2}+\frac{1}{\sin (\theta)^{2}} \xi_{\phi}^{2}+\xi_{\theta}^{2}\right]
$$

It obviously satisfies the first two ellipticity conditions

$$
\begin{gathered}
\sigma_{\psi}^{2}\left(\mathfrak{h}_{\text {lin }}-\varepsilon\right)\left(r, \phi, \theta, \tau, \xi_{\phi}, \xi_{\theta}\right) \neq 0 \text { for all }\left(r, \phi, \theta, \tau, \xi_{\phi}, \xi_{\theta}\right) \in T^{*}\left(\mathbb{R}_{+} \times S^{2}\right) \backslash 0 \\
r^{2} \sigma_{\psi}^{2}\left(\mathfrak{h}_{\text {lin }}-\varepsilon\right)\left(r, \phi, \theta, r^{-1} \tau, \xi_{\phi}, \xi_{\theta}\right) \neq 0 \text { for all }\left(r, \phi, \theta, \tau, \xi_{\phi}, \xi_{\theta}\right) \in T^{*}\left(\overline{\mathbb{R}}_{+} \times S^{2}\right) \backslash 0
\end{gathered}
$$


(ii) The conormal symbol, with $z \in \mathbb{C}$, is given by

$$
\sigma_{M}^{2}\left(\mathfrak{h}_{\operatorname{lin}}-\varepsilon\right)(z)=\frac{1}{2}\left[\mathfrak{l}^{2}-z^{2}+z\right]
$$

and represents a family of continous operators

$$
\sigma_{M}^{2}\left(\mathfrak{h}_{\operatorname{lin}}-\varepsilon\right)(z): H^{s}\left(S^{2}\right) \rightarrow H^{s-2}\left(S^{2}\right) .
$$

It follows from the basic properties of the total angular momentum operator $\mathfrak{l}^{2}$, cf. Ref. [12], that the operator family (2.4) has a pure point spectrum with eigenfunctions $Y_{l m} \in H^{\infty}\left(S^{2}\right)$ and eigenvalues $\frac{1}{2}\left(l(l+1)-z^{2}+z\right), l \in \mathbb{Z}_{+}$. The second ellipticity condition requires that the operators (2.4) are isomorphisms for some $s$ and all $z \in \Gamma_{3 / 2-\gamma}$, where $\Gamma_{3 / 2-\gamma}$ is a straight line through $3 / 2-\gamma$ parallel to the imaginary axis. This condition is obviously satisfied for $s \in \mathbb{R}$ and $3 / 2-\gamma \notin \mathbb{Z}$.

(iii) The principal exit symbols, expressed in cartesian coordinates, are given by

$$
\begin{gathered}
\sigma_{e}^{0}\left(\mathfrak{h}_{\text {lin }}-\varepsilon\right)=\frac{1}{2} \sum_{i=1}^{3} \xi_{i}^{2}-\varepsilon>0 \text { for all }(x, \xi) \in T^{*} X^{\wedge}, \varepsilon<0 \\
\sigma_{\psi, e}^{2,0}\left(\mathfrak{h}_{\text {lin }}-\varepsilon\right)=\frac{1}{2} \sum_{i=1}^{3} \xi_{i}^{2}>0 \text { for all }(x, \xi) \in T^{*} X^{\wedge} \backslash 0
\end{gathered}
$$

and satisfy the third ellipticity condition.

It turns out that the Hartree potential $V_{H}$ can be attached to the linear part of the Fock operator without affecting the assertions of Proposition 2. For this, we first prove two lemmas concerning Coulomb convolutions on weighted Sobolev spaces with asymptotics.

Lemma 1. Suppose $u \in \mathcal{S}_{P}^{\gamma}$ for some asymptotic type $P$ and weight data $(\gamma, \Theta)$ with $\gamma<3 / 2$, $\Theta=(-\infty, 0]$. The convolution product $\omega(x) \frac{1}{|x|} * u$, with arbitrary cut-off function $\omega$, belongs to $\mathcal{S}_{Q}^{\gamma}$ for some asymptotic type $Q$.

Proof. Given an appropriate cut-off function $\eta$, with $\eta(x)=1$ for $x \in \operatorname{supp} \omega, u$ can be written as $u=\eta u+(1-\eta) u$, where the lemma is obviously true for the second part of the decomposition. For the first part, we use the partial wave expansion of the Coulomb potential

$$
\frac{1}{|x-y|}=\sum_{l=0}^{\infty} \frac{4 \pi}{2 l+1} \frac{r_{<}^{l}}{r_{>}^{l+1}} \sum_{m} Y_{l, m}(\hat{x}) Y_{l, m}^{*}(\hat{y}),
$$

with $r_{<}:=\min \{|x|,|y|\}, r_{>}:=\max \{|x|,|y|\}$, and $\hat{x}:=x /|x|$, in order to study the effect of the convolution

$$
\int_{\mathbb{R}^{3}} \frac{1}{|x-y|} c(\hat{y})|y|^{-p} \ln ^{k}|y| \eta(|y|) d y,
$$

on individual terms of the asymptotic expansion. For $c(\hat{x}) \in C^{\infty}\left(S^{2}\right)$, the expansion in spherical harmonics

$$
c(\hat{x})=\sum_{l=0}^{\infty} \sum_{m} c_{l, m} Y_{l, m}(\hat{x})
$$


is absolutely convergent. Inserting the expansion (2.5) into the integral (2.6) we obtain

$$
\sum_{l=0}^{\infty} \frac{4 \pi}{2 l+1}\left(\sum_{m} c_{l, m} Y_{l, m}(\hat{x})\right)\left[\frac{1}{|x|^{l+1}} \int_{0}^{|x|} r^{l-p+2} \ln ^{k} r d r+|x|^{l} \int_{|x|}^{\infty} r^{1-l-p} \ln ^{k} r \eta(r) d r\right],
$$

where we assume $|x|<\epsilon$. After some rearrangements, the integral (2.6) becomes

$$
\begin{gathered}
|x|^{2-p} b_{0}(\hat{x})+g_{0}(x) \text { for } k=0, \\
|x|^{2-p} \sum_{j=0}^{k+1} b_{j}(\hat{x}) \ln |x|^{j}+g_{k}(x) \text { for } k>0
\end{gathered}
$$

with

$$
\begin{gathered}
b_{j}(\hat{x})=\sum_{l=0}^{\infty}{ }^{\prime} \frac{4 \pi}{2 l+1} a_{l, j}\left(\sum_{m} c_{l, m} Y_{l, m}(\hat{x})\right) \text { for } j<k+1, \\
b_{k+1}(\hat{x})=\frac{4 \pi}{3-2 p} a_{2-p, k+1}\left(\sum_{m} c_{2-p, m} Y_{2-p, m}(\hat{x})\right),
\end{gathered}
$$

where the constants $a_{l, j}$ satisfy the estimate $\left|a_{l, j}\right| \lesssim l^{-1}$, and $g_{k} \in C^{\infty}\left(\mathbb{R}^{3}\right)$. The prime in $(2.7)$ indicates that the sum runs over all $l$ except $l=2-p$.

Lemma 2. Suppose $u \in \mathcal{S}_{P}^{\gamma}$ for some asymptotic type $P$ and weight data $(\gamma, \Theta)$ with $\gamma<3 / 2$, $\Theta=(-\infty, 0]$. The convolution product $\frac{1}{|x|} * u$ satisfies the asymptotic estimates

$$
(1-\omega(x))\left|\partial^{\alpha} \frac{1}{|x|} * u\right| \lesssim(1+|x|)^{-1-|\alpha|} \text { for all }|\alpha| \geq 0 .
$$

Proof. Given an approriate cut-off function $\eta, u$ can be written as $u=\eta u+(1-\eta) u$, where the lemma is clearly true for the first term of the decomposition. We consider the expansion of $(1-\eta) u$ in spherical harmonics

$$
(1-\eta) u(x)=\sum_{l} \sum_{m} Y_{l, m}(\hat{x}) u_{l, m}(|x|) .
$$

Using the partial wave expansion of the Coulomb potential (2.5), the convolution product can be written as

$$
\frac{1}{|x|} *(1-\eta) u=\sum_{l} \frac{4 \pi}{2 l+1} \sum_{m} Y_{l, m}(\hat{x}) v_{l, m}(|x|)
$$

with

$$
v_{l, m}(|x|)=\frac{1}{|x|^{l+1}} \int_{0}^{|x|} r^{l+2} u_{l, m}(r) d r+|x|^{l} \int_{|x|}^{\infty} r^{1-l} u_{l, m}(r) d r .
$$

It is therefore sufficient to prove estimates $\left|v_{l, m}^{(n)}(|x|)\right| \lesssim(1+|x|)^{-1-l-n}$ for the $n^{\prime}$ th derivates $v_{l, m}^{(n)}$. Because of $(1-\eta) u \in \mathcal{S}\left(\mathbb{R}, C^{\infty}\left(S^{2}\right)\right)_{\mathbb{R}_{+} \times S^{2}}$, the second integral vanishes for $|x| \rightarrow \infty$ faster than any $|x|^{-n}$ for $n \in \mathbb{Z}_{+}$and $v_{l, m}(|x|) \lesssim(1+|x|)^{-l-1}$. For the same reason, the first derivative

$$
v_{l, m}^{(1)}(|x|)=-\frac{l+1}{|x|^{l+2}} \int_{0}^{|x|} r^{l+2} u_{l, m}(r) d r+l|x|^{l-1} \int_{|x|}^{\infty} r^{1-l} u_{l, m}(r) d r .
$$

satisfies the estimate $\left|v_{l, m}^{(1)}(|x|)\right| \lesssim(1+|x|)^{-l-2}$. This argument can be continued to arbitrarily high derivatives of $v_{l, m}$. 
Lemma 3. The Hartree potential $V_{H}$ for a set $\left\{\phi_{i}\right\}_{i=1, N / 2}$ of asymptotically well behaved functions belongs to $C^{\infty}\left(\overline{\mathbb{R}}_{+} \times S^{2}\right)$. Furthermore it satisfies the estimate

$$
(1-\omega(x))\left|\partial^{\alpha} V_{H}(x)\right| \lesssim(1+|x|)^{-1-|\alpha|} \text { for all }|\alpha| \geq 0 .
$$

Proof. This is an immediate consequence of Remark 1 and Lemmas 1, 2.

Lemma 4. Given a set $\left\{\phi_{i}\right\}_{i=1, N / 2}$ of asymptotically well behaved functions. The corresponding local part of the shifted Fock operator

$$
\mathfrak{h}_{l o c}-\varepsilon:=\mathfrak{h}_{\text {lin }}+V_{H}-b \mathcal{P}-\varepsilon \text { for } \varepsilon<0,
$$

belongs to $C^{2}\left(X^{\wedge}, \underline{g}\right)$ and is elliptic, in the sense of Def. 6, p. 259 of Ref. [3], for weight data $g=(\gamma, \gamma-2, \Theta)$ with $3 / 2-\gamma \in \mathbb{R} \backslash \mathbb{Z}, \gamma<3 / 2$ and $\Theta=(-\infty, 0]$. There exists a parametrix $\overline{\mathcal{P}}_{\text {loc }} \in C^{-2}\left(X^{\wedge}, \underline{h}\right)$ for $\underline{h}=(\gamma-2, \gamma, \Theta)$ and a corresponding Green operator $\mathcal{G}_{\text {loc }} \in C_{G}\left(X^{\wedge}, \underline{m}\right)$ for $\underline{m}=(\gamma, \gamma, \Theta)$ with

$$
\mathcal{P}_{l o c}\left(\mathfrak{h}_{l o c}-\varepsilon\right)=I+\mathcal{G}_{l o c}
$$

which provide continous maps

$$
\mathcal{P}_{l o c}: \mathcal{K}_{P}^{s, \gamma}\left(X^{\wedge}\right) \longrightarrow \mathcal{K}_{Q}^{s+2, \gamma+2}\left(X^{\wedge}\right) \text { and } \mathcal{G}_{l o c}: \mathcal{K}^{s, \gamma}\left(X^{\wedge}\right) \longrightarrow \mathcal{S}_{Q}^{\gamma}\left(X^{\wedge}\right)
$$

Proof. This is an immediate consequence of Proposition 2 and Lemma 3, where the latter ensures that the exit part of the symbol belongs to $S^{2,0}\left(\mathbb{R}^{3} \times \mathbb{R}^{3}\right)$, cf. Section 3.2. The existence of a parametrix for elliptic operators has been proven e.g. in Theorem 8, p. 259 of Ref. [3].

\subsection{Proof of Theorem 1}

Proof. The shifted HF equation at the $n$ 'th iteration of the level-shifting algorithm (2.1) is given by

$$
\left(\mathfrak{h}_{\text {lin }}+V_{H}^{(n-1)}+\mathcal{U}^{(n-1)}-b \mathcal{P}^{(n-1)}\right) \phi_{i}^{(n)}=\varepsilon_{i}^{(n)} \phi_{i}^{(n)} .
$$

For sufficiently large parameter $b, N / 2$ negative eigenvalues $\varepsilon_{i}^{(n)}$ are known to exist, cf. Ref. [2]. According to Lemma 4, we can separate the local part of the Fock operator and obtain

$$
\left(\mathfrak{h}_{\mathrm{loc}}^{(n-1)}-\varepsilon_{i}^{(n)}\right) \phi_{i}^{(n)}=-\mathcal{U}^{(n-1)} \phi_{i}^{(n)}
$$

where the term in parenthesis corresponds to an elliptic operator in $C^{2}\left(X^{\wedge}, \underline{g}\right)$. Acting with the parametrix $\mathcal{P}_{\text {loc }}$ on the left of (2.10) yields

$$
\phi_{i}^{(n)}=-\mathcal{P}_{\mathrm{loc}} \mathcal{U}^{(n-1)} \phi_{i}^{(n)}-\mathcal{G}_{\mathrm{loc}} \phi_{i}^{(n)} .
$$

With this equation at hand, we can perform a bootstrap argument for which we introduce a sequence of weighted Sobolev spaces $\mathcal{K}_{P_{k}}^{k, \gamma}\left(X^{\wedge}\right), k=1,2, \ldots$, with weight data $(\gamma,(\gamma-k-1,0])$. Suppose $\phi_{i}^{(n)} \in \mathcal{K}_{P_{k}}^{k, \gamma}\left(X^{\wedge}\right)$, and let us further assume that $\mathcal{U}^{(n-1)} \phi_{i}^{(n)}$ belongs to $\mathcal{K}_{P_{k}}^{k, \gamma}\left(X^{\wedge}\right)$, it follows from (2.11) that $\phi_{i}^{(n)}$ belongs to $\mathcal{K}_{P_{k+2}}^{k+2, \gamma}\left(X^{\wedge}\right)$.

From the work of Lieb, Simon and Lions $[8,9]$ it is known that the eigenfunctions $\phi_{i}^{(n)}$ are globally Lipschitz and decay exponentially for $r \rightarrow \infty$. According to the definitions (1.9) and (1.10), these eigenfunctions, therefore, belong to $\mathcal{K}^{0, \gamma}\left(X^{\wedge}\right)$ for $\gamma<3 / 2$. As a starting point for the bootstrap argument we choose $\mathcal{K}^{0,0}\left(X^{\wedge}\right) \equiv L^{2}\left(\mathbb{R}^{3}\right)$. It follows from $(2.11)$ that $\phi_{i}^{(n)}$ belongs to $\mathcal{K}_{P_{1}^{1, \gamma}}\left(X^{\wedge}\right)$ if 
$\mathcal{U}^{(n-1)} \phi_{i}^{(n)}$ belongs to $\mathcal{K}^{0,0}\left(X^{\wedge}\right)$. In order to deal with convolutions of the Coulomb potential, the following estimate (cf. Ref. [7])

$$
\left|\frac{1}{|x|} *(f g)\right| \leq\left\|\frac{f(\cdot)}{|x-\cdot|}\right\|_{L^{2}\left(\mathbb{R}^{3}\right)}\|g\|_{\left.L^{2} \mathbb{R}^{3}\right)} \lesssim\|f\|_{H^{1}\left(\mathbb{R}^{3}\right)}\|g\|_{L^{2}\left(\mathbb{R}^{3}\right)}
$$

uniform in $x$, with $f \in H^{1}\left(\mathbb{R}^{3}\right), g \in L^{2}\left(\mathbb{R}^{3}\right)$, turned out to be useful. From this estimate, and $\phi_{j}^{(n-1)} \in H^{1}\left(\mathbb{R}^{3}\right)$ (cf. proof of Proposition 1), we obtain the desired result

$$
\left\|\phi_{j}^{(n-1)} \frac{1}{|x|} *\left(\phi_{j}^{(n-1)} \phi_{i}^{(n)}\right)\right\|_{\mathcal{K}^{0,0}\left(X^{\wedge}\right)} \lesssim\left\|\phi_{i}^{(n)}\right\|_{\mathcal{K}^{0,0}\left(X^{\wedge}\right)}\left\|\phi_{j}^{(n-1)}\right\|_{H^{1}\left(\mathbb{R}^{3}\right)}\left\|\phi_{j}^{(n-1)}\right\|_{\mathcal{K}^{0,0}\left(X^{\wedge}\right)},
$$

for the individual terms of $\mathcal{U}^{(n-1)} \phi_{i}^{(n)}$.

In the next step we proceed to weighted Sobolev spaces with asymptotics, where it has to be shown that $\mathcal{U}^{(n-1)} \phi_{i}^{(n)}$ belongs to $\mathcal{K}_{P_{k}}^{k, \gamma}\left(X^{\wedge}\right)$. Let us first point out that the products $\phi_{i}^{(n)} \phi_{j}^{(n-1)}$ with $\phi_{i}^{(n)} \in \mathcal{K}_{P_{k}}^{k, \gamma}\left(X^{\wedge}\right)$ and $\phi_{j}^{(n-1)} \in \mathcal{S}_{P}^{\gamma}\left(X^{\wedge}\right)$ belong to $\mathcal{K}_{P_{k}}^{k, \gamma}\left(X^{\wedge}\right)$ again. Here we assume that the asymptotic type $P_{k}$ has been chosen broad enough to comprise these products. Again, we consider individual terms

$$
\phi_{j}^{(n-1)} \frac{1}{|x|} *\left(\phi_{j}^{(n-1)} \phi_{i}^{(n)}\right) \text {, }
$$

separately and perform the decomposition of $\phi_{j}^{(n-1)}=v_{j}^{(n-1)}+w_{j}^{(n-1)}$ and $\phi_{i}^{(n)}=v_{i}^{(n)}+w_{i}^{(n)}$ into its components in $\mathcal{E}_{P_{k}}^{\gamma}\left(X^{\wedge}\right)$ and $\mathcal{K}_{\Theta_{k}}^{k, \gamma}\left(X^{\wedge}\right)$, where we assume w.l.o.g. that $v_{j}^{(n-1)}$ and $v_{i}^{(n)}$ have compact support within in a bounded neighbourhood of the origin $\Omega$. Correspondingly, we decompose the quadratic term

$$
\phi_{j}^{(n-1)} \phi_{i}^{(n)}=v_{c}+w_{c}
$$

with $v_{c}:=v_{j}^{(n-1)} v_{i}^{(n)} \in \mathcal{K}_{P_{k}}^{\infty, \gamma}\left(X^{\wedge}\right)$ and $w_{c}:=v_{j}^{(n-1)} w_{i}^{(n)}+w_{j}^{(n-1)} v_{i}^{(n)}+w_{j}^{(n-1)} w_{i}^{(n)} \in \mathcal{K}_{\Theta_{k}}^{k, \gamma}\left(X^{\wedge}\right)$. Then it follows from Lemmas 1 and 2 that

$$
\left\|v_{j}^{(n-1)} \frac{1}{|x|} * v_{c}\right\|_{\mathcal{K}_{P_{k}}^{k, \gamma}\left(X^{\wedge}\right)}<\infty .
$$

The second and third combination

$$
w_{j}^{(n-1)} \frac{1}{|x|} * v_{c}, w_{j}^{(n-1)} \frac{1}{|x|} * w_{c},
$$

are supposed to belong to $\mathcal{K}_{\Theta_{k}}^{k, \gamma}\left(X^{\wedge}\right)$ which can be proven by analogous arguments. For the second combination we introduce a cut-off function $\eta$ with $\operatorname{supp} \eta \subset \Omega$ according to (1.10) and consider first the weighted Sobolev space $\mathcal{H}^{k, k+1-\epsilon}\left(X^{\wedge}\right)$ for $1>\epsilon>0$ where we get

$$
\begin{aligned}
\left\|\eta w_{j}^{(n-1)} \frac{1}{|x|} * v_{c}\right\|_{\mathcal{H}^{k, k+1-\epsilon\left(X^{\wedge}\right)}} \lesssim \sum_{|\alpha| \leq k} \sum_{\alpha_{1}+\alpha_{2}=\alpha}\left\||x|^{\left|\alpha_{1}\right|} \partial^{\alpha_{1}} \frac{1}{|x|} * v_{c}\right\|_{L^{\infty}(\Omega)} \\
\times\left\||x|^{-k-1+\epsilon+\left|\alpha_{2}\right|} \partial^{\alpha_{2}}\left(\eta w_{j}^{(n-1)}\right)\right\|_{L^{2}(\Omega)} \lesssim\left\|\eta w_{j}^{(n-1)}\right\|_{\mathcal{H}^{k, k+1-\epsilon\left(X^{\wedge}\right)}}<\infty .
\end{aligned}
$$

The last step is a consequence of Lemma 1 which demonstrates boundedness of the factors in $L^{\infty}$ norm. A similar estimate can be obtained for the $(1-\eta)(\cdot)$ term in $H^{k}\left(\mathbb{R}^{3}\right)$. The third combination follows by similar estimates, except that the $L^{\infty}$ terms need special considerations. In order to keep 
the asymptotic behaviour of the convolution under control, we first split the Coulomb potential into $L^{2}\left(\mathbb{R}^{3}\right)+L^{\infty}\left(\mathbb{R}^{3}\right)$ through the decomposition

$$
\frac{1}{|x|}=\mathcal{Y}(x)+\mathcal{W}(x), \quad \text { with } \mathcal{Y}(x):=\frac{e^{-|x|}}{|x|} \text { and } \mathcal{W}(x):=\frac{1-e^{-|x|}}{|x|}
$$

For the Yukawa part of the potential, we obtain, by applying Young's inequality, the estimate

$$
\begin{aligned}
\left\|\mathcal{Y} * \partial^{\alpha_{1}} w_{c}\right\|_{L^{\infty}(\Omega)} & \leq\|\mathcal{Y}\|_{L^{2}\left(\mathbb{R}^{3}\right)}\left\|\partial^{\alpha_{1}} w_{c}\right\|_{L^{2}\left(\mathbb{R}^{3}\right)} \\
& \lesssim\left\||x|^{-k-1+\epsilon+\left|\alpha_{1}\right|} \partial^{\alpha_{1}}\left(\tilde{\eta} w_{c}\right)\right\|_{L^{2}\left(\mathbb{R}^{3}\right)}+\left\|\partial^{\alpha_{1}}\left((1-\tilde{\eta}) w_{c}\right)\right\|_{L^{2}\left(\mathbb{R}^{3}\right)} \\
& \lesssim\left\|w_{c}\right\|_{\mathcal{K}^{k, k+1-\epsilon}\left(X^{\wedge}\right)},
\end{aligned}
$$

where in the second last step $w_{c}$ has been split up according to (1.10) using an arbitrary cut-off function $\tilde{\eta}$. Using another variant of Young's inequality, the bounded part of the potential can be estimated

$$
\begin{aligned}
\left\|\mathcal{W} * \partial^{\alpha_{1}} w_{c}\right\|_{L^{\infty}(\Omega)} & \leq\|\mathcal{W}\|_{L^{\infty}\left(\mathbb{R}^{3}\right)}\left\|\partial^{\alpha_{1}} w_{c}\right\|_{L^{1}\left(\mathbb{R}^{3}\right)} \\
& \lesssim\left\||x|^{-\gamma-k-1+\epsilon+\left|\alpha_{1}\right|} \partial^{\alpha_{1}}\left(v_{j}^{(n-1)} w_{i}^{(n)}+w_{j}^{(n-1)} v_{i}^{(n)}\right)\right\|_{L^{1}(\Omega)} \\
& +\left\|\partial^{\alpha_{1}}\left(w_{j}^{(n-1)} w_{i}^{(n)}\right)\right\|_{L^{1}\left(\mathbb{R}^{3}\right)} \\
& \lesssim\left\|v_{j}^{(n-1)}\right\|_{\mathcal{K}_{P_{k}}^{k, \gamma}\left(X^{\wedge}\right)}\left\|w_{i}^{(n)}\right\|_{\mathcal{K}^{k, k+1-\epsilon}\left(X^{\wedge}\right)}+\left\|w_{j}^{(n-1)}\right\|_{\mathcal{K}^{k, k+1-\epsilon}\left(X^{\wedge}\right)}\left\|v_{i}^{(n)}\right\|_{\mathcal{K}_{P_{k}}^{k, \gamma}\left(X^{\wedge}\right)} \\
& +\left\|w_{j}^{(n-1)}\right\|_{\mathcal{K}^{k, k+1-\epsilon}\left(X^{\wedge}\right)}\left\|w_{i}^{(n)}\right\|_{\mathcal{K}^{k, k+1-\epsilon}\left(X^{\wedge}\right)},
\end{aligned}
$$

where we assume $-k-1+\epsilon \leq \gamma<3 / 2$.

It remains to consider the fourth combination

$$
v_{j}^{(n-1)} \frac{1}{|x|} * w_{c}
$$

where we decompose the convolution term via a Taylor series

$$
\frac{1}{|x|} * w_{c}=\sum_{|\beta| \leq k-1} c_{\beta} x^{\beta}+W_{k}, \text { with } c_{\beta}:=\frac{1}{\beta !} \int \frac{\partial^{\beta} w_{c}(y)}{|y|} d y
$$

and formal residuum

$$
W_{k}:=\frac{1}{|x|} * w_{c}-\sum_{|\beta| \leq k-1} c_{\beta} x^{\beta},
$$

which makes sense because of $(2.14),(2.15)$ and the compact supports of the functions $v_{j}^{(n-1)}$. For the terms in the Taylor series we get

$$
\left\|v_{j}^{(n-1)} x^{\beta}\right\|_{\mathcal{K}_{P_{k}}^{k, \gamma}}<\infty \text { with }|\beta| \leq k-1 .
$$

The derivatives of the residuum $W_{k}$ can be estimated according to

$$
\begin{aligned}
\left|\partial^{\alpha} W_{k}\right| & =\left|\frac{1}{|x|} * \partial^{\alpha} w_{c}-\sum_{0 \leq|\beta| \leq k-1-|\alpha|} \frac{x^{\beta}}{\beta !} \int \frac{\partial^{\alpha+\beta} w_{c}(y)}{|y|} d y\right| \\
& =\left|\sum_{|\beta|=k-|\alpha|} \frac{x^{\beta}}{\beta !} \int \frac{\partial^{\alpha+\beta} w_{c}(y)}{|t x-y|} d y\right| \text { with } t \in[0,1] \\
& \lesssim|x|^{k-|\alpha|} \sum_{|\beta|=k-|\alpha|}\left(\left\|\mathcal{Y} * \partial^{\alpha+\beta} w_{c}\right\|_{L^{\infty}(\Omega)}+\left\|\mathcal{W} * \partial^{\alpha+\beta} w_{c}\right\|_{L^{\infty}(\Omega)}\right)
\end{aligned}
$$


using the integral representation for the remainder of the Taylor expansion together with (2.14) and (2.15). As already mentioned above, the fourth combination (2.16) has compact support within a bounded neighbourhood $\Omega$ of the origin. It is therefore sufficient to consider for the residuum the space $\mathcal{H}^{k, k+1-\epsilon}\left(X^{\wedge}\right)$ where the estimate

$$
\begin{aligned}
\left\|v_{j}^{(n-1)} W_{k}\right\|_{\mathcal{H}^{k, k+1-\epsilon}\left(X^{\wedge}\right)} \lesssim \sum_{\left|\alpha_{1}\right|+\left|\alpha_{2}\right| \leq k}\left\|\left.|| x\right|^{-k-1+\epsilon+\left|\alpha_{1}\right|+\left|\alpha_{2}\right|} \partial^{\alpha_{1}} v_{j}^{(n-1)} \partial^{\alpha_{2}} W_{k}\right\|_{L^{2}(\Omega)} \\
\lesssim \sum_{\left|\alpha_{1}\right|+\left|\alpha_{2}\right| \leq k}\left\||x|^{-1+\epsilon+\left|\alpha_{1}\right|} \partial^{\alpha_{1}} v_{j}^{(n-1)}\right\|_{L^{2}(\Omega)}\left\||x|^{-k+\left|\alpha_{2}\right|} \partial^{\alpha_{2}} W_{k}\right\|_{L^{\infty}(\Omega)}<\infty(2.1
\end{aligned}
$$

completes the proof that $\mathcal{U}^{(n-1)} \phi_{i}^{(n)}$ belongs to $\mathcal{K}_{P_{k}}^{k, \gamma}\left(X^{\wedge}\right)$.

After having shown that $\phi_{i}^{(n)} \in \mathcal{K}_{P_{\infty}}^{\infty, \gamma}\left(X^{\wedge}\right)$, it follows immediately that $\phi_{j}^{(n-1)} \phi_{i}^{(n)}$ belong to a function space $\mathcal{S}_{Q}^{\gamma}$. Together with Lemmas 1 and 2 we can further conclude that $\mathcal{U}^{(n-1)} \phi_{i}^{(n)}$ belongs to some $\mathcal{S}_{Q}^{\gamma}$. Using the canonical decomposition of the parametrix (cf. Ref. [3])

$$
\mathcal{P}=\mathcal{P}^{\prime}+\tilde{\mathcal{P}}+\tilde{\mathcal{G}} \text { with } \mathcal{P}^{\prime}:=\eta \mathcal{P}_{0} \tilde{\eta} \text { and } \tilde{\mathcal{P}}:=(1-\eta) \mathcal{P}_{1}(1-\tilde{\tilde{\eta}}),
$$

with cut-off functions $\tilde{\eta} \equiv 1$ on $\operatorname{supp} \eta, \eta \equiv 1$ on supp $\tilde{\tilde{\eta}}$, where $\mathcal{P}_{1}$ corresponds to a pseudo-differential operator on $\mathbb{R}^{3}$ with symbol in $S^{-2,0}\left(\mathbb{R}^{3} \times \mathbb{R}^{3}\right.$ ) (cf. Section 3.2 ), which maps $\mathcal{S}\left(\mathbb{R}^{3}\right)$ into $\mathcal{S}\left(\mathbb{R}^{3}\right)$, we obtain from $(2.11)$ that $\phi_{i}^{(n)}$ itself belongs to a function space $\mathcal{S}_{Q}^{\gamma}$.

It remains to specify the asymptotic type $Q$. This can be done by inserting the asymptotic expansion of the eigenfunctions

$$
\phi_{i}^{(n)}=\omega \sum_{j \in \mathbb{Z}_{+}} \sum_{k=0}^{m_{j}}\left(\sum_{l, m} c_{j, k, l m} Y_{l m}\right) r^{-p_{j}} \ln ^{k} r+\Phi
$$

into the shifted HF equation (2.9) and comparing different terms $Y_{l m}(\phi, \theta) r^{-p_{j}} \ln ^{k} r$. By definition, we assume for $k=m_{j}$ that there exists a $c_{j, m_{j}, l m} \neq 0$ for some $l \geq 0$ and $-l \leq m \leq l$. The action of the Laplacian on these terms in a neighbourhood of the origin is given by

$$
\begin{aligned}
& \frac{1}{r^{2}}\left[\left(-r \frac{\partial}{\partial r}\right)^{2}-\left(-r \frac{\partial}{\partial r}\right)-\mathfrak{l}^{2}\right] Y_{l m} r^{-p_{j}} \ln ^{k} r \\
& \quad=Y_{l m} r^{-p_{j}-2}\left[\left(p_{j}\left(p_{j}-1\right)-l(l+1)\right) \ln ^{k} r-k\left(2 p_{j}-1\right) \ln ^{k-1} r+k(k-1) \ln ^{k-2} r\right] .
\end{aligned}
$$

Furthermore we have to consider the action of local potentials which can be easily derived from their asymptotic expansions

$$
-\frac{Z}{r}+V_{\mathrm{ext}}+V_{H}^{(n-1)}-\varepsilon^{(n)} \sim-\frac{Z}{r}+\sum_{n \in \mathbb{Z}_{+}}\left(\sum_{l=0}^{n} \sum_{m} d_{n, l m} Y_{l m}\right) r^{n},
$$

and of the nonlocal exchange and projection operators

$$
\begin{gathered}
\mathcal{U}^{(n-1)} Y_{l m} r^{-p_{j}} \ln ^{k} r \sim Y_{l m} r^{2-p_{j}}\left(\sum_{s=0}^{k} d_{s} \ln ^{s} r+\delta_{l, 2-p_{j}} d_{k+1} \ln ^{k+1} r\right)+d_{\infty} r^{l} Y_{l m}+\cdots \\
\mathcal{P}^{(n-1)} Y_{l m} r^{-p_{j}} \ln ^{k} r \sim Y_{l m} \sum_{l \leq n} d_{n} r^{n}
\end{gathered}
$$

which follow immediately from Definition 1 and the proof of Lemma 1. 
At first we want to consider the cases $-p_{j} \notin \mathbb{Z}_{+}$. The proof proceeds in an inductive manner starting from the interval $0<-p_{j}<1$. According to (2.20), the Laplacian maps

$$
\Delta:-p_{j} \in(0,1) \longrightarrow-p_{j}-2 \in(-2,-1) .
$$

These terms have no counterparts in (2.9) and must therefore satisfy the equation

$$
p_{j}\left(p_{j}-1\right)-l(l+1)=0 \text { with } l \in \mathbb{Z}_{+},
$$

for the highest power $m_{j}$ of the logarithm. This leads to a contradiction because (2.24) has only integer solutions. Proceeding to the next interval $1<-p_{j}<2$, the Laplacian (2.20) maps

$$
\Delta:-p_{j} \in(1,2) \longrightarrow-p_{j}-2 \in(-1,0) .
$$

Possible counterterms originating from the asymptotic expansion of the local potentials $(2.21)$

$$
r^{-1}:-p_{j} \in(0,1) \nrightarrow-p_{j}-1 \in(-1,0)
$$

cannot contribute because we have already shown that there exists no $0<-p_{j}<1$. Therefore we obtain again a contradiction from (2.24). A similar argument can be applied to the interval $2<-p_{j}<3$, where

$$
\Delta:-p_{j} \in(2,3) \longrightarrow-p_{j}-2 \in(0,1),
$$

and possible counterterms from the local potentials $(2.21)$

$$
\begin{gathered}
r^{-1}:-p_{j} \in(1,2) \nrightarrow-p_{j}-1 \in(0,1) \\
r^{0}:-p_{j} \in(0,1) \nrightarrow-p_{j} \in(0,1)
\end{gathered}
$$

are again excluded because there exists no $0<-p_{j} \neq 1<2$. The inductive arguments can be extended to arbitrary intervals $(n, n+1)$ with $n \in \mathbb{Z}_{+}$which proves that $-p_{j} \in \mathbb{Z}_{+}$for all $j$.

It remains to study the cases $-p_{j} \in \mathbb{Z}_{+}$with $m_{j} \geq 0$. Like in the first part of the proof, we consider the action of the Laplacian on terms with $p_{0}=0$ and continue in an inductive manner. Suppose $k=m_{0}$, the leading order term $r^{-2} \ln ^{m_{0}} r$ then yields $c_{0, m_{0}, l m}=0$ for $l \neq 0$. For $l=0$ and $m_{0}>0$, we obtain $c_{0, m_{0}, 00}=0$ from the logarithmic term $r^{-2} \ln ^{m_{0}-1} r$. This demonstrates that all coefficients, except $c_{0,0,00}$, must vanish. Furthermore, we observe that the asymptotic expansions (2.21), (2.22) and (2.23) acting on $c_{0,0,00} Y_{00}$ contribute terms of the form

$$
r^{-1} Y_{00}, \text { and } r^{n} Y_{l m} \text { with } n \in \mathbb{Z}_{+}, l \leq n
$$

The next terms in the series with $-p_{1}=1$ can be treated in a similar manner. Suppose first $k=m_{1}>0$, then (2.20) in leading order $r^{-1} \ln ^{m_{1}} r$ yields $c_{1, m_{1}, l m}=0$ for $l \neq 1$. From the term $r^{-1} \ln ^{m_{1}-1} r$, we obtain $c_{1, m_{1}, 1 m}=0$ because no other terms from (2.25) contribute. The possibly only nonvanishing coefficients are $c_{1,0,00}$ (linked with $c_{0,0,00} r^{-1} Y_{00}$ ) and $c_{1,0,1 m}$. Again, we observe that the asymptotic expansions (2.21), (2.22) and (2.23) acting on $c_{1,0,00} r Y_{00}$ and $c_{1,0,1 m} r Y_{1 m}$ contribute terms of the form

$$
Y_{00}, Y_{1 m}, \text { and } r^{n+1} Y_{l m} \text { with } n \in \mathbb{Z}_{+}, l \leq n+1 .
$$

This line of arguments can be continued to arbitrary terms with $-p_{j}=j$, where $(2.20)$ with $k=$ $m_{j}>0$ in leading order $r^{j-2} \ln ^{m_{j}} r$ yields $c_{j, m_{j}, l m}=0$ for $l \neq j$. As before, the next lower logarithmic term $r^{j-2} \ln ^{m_{j}-1} r$ yields $c_{j, m_{j}, j m}=0$ because no other terms of this form are contained in (2.25), (2.26), etc.. Therefore, we obtain $m_{j}=0$ and possibly nonvanishing coefficients $c_{j, 0, l m}$ with $l \leq j$ (coefficients with $l<j$ are linked in leading order with correspoding terms from (2.25), (2.26), etc.). 
The terms $c_{j, 0, l m} r^{j} Y_{l m}$ couple with the asymptotic expansions (2.21), (2.22) and (2.23) to generate terms of the form

$$
{ }^{j-1} Y_{l m} \text { with } l \leq j \text {, and }{ }^{n+j} Y_{l m} \text { with } n \in \mathbb{Z}_{+}, l \leq n+j .
$$

These arguments demonstrate the absence of logarithmic terms in the expansion (2.19). Furthermore our arguments show that for each $j$ only a finite number of spherical harmonics with $l \leq-p_{j}$ contribute.

\subsection{Proof of Theorem 2}

Proof. In order to prove that the self-consistent-field solutions of the Hartree-Fock equation obtained via the level-shifting algorithm are asymptotically well behaved, we proceed in two steps. First we show via a bootstrap argument, similar to which we have used in the proof of Theorem 1, that these solutions belong to $\mathcal{K}_{P}^{\infty, \gamma}\left(X^{\wedge}\right)$ and restrict in the following the function space further to $\mathcal{S}_{P}^{\gamma}\left(X^{\wedge}\right)$.

The Fock operator of the $n$ 'th iteration splits up into

$$
\mathfrak{h}^{(n-1)}=\mathfrak{h}_{\text {lin }}+V^{(n-1)}, \quad \text { with } V^{(n-1)}:=V_{H}^{(n-1)}+\mathcal{U}^{(n-1)}-b \mathcal{P}^{(n-1)},
$$

where the shifted linear part $\mathfrak{h}_{\text {lin }}-\varepsilon$, with $\varepsilon<0$, corresponds to an elliptic operator in $C^{2}\left(X^{\wedge}, \underline{g}\right)$. According to Proposition 2, there exists a parametrix $\mathcal{P}_{\text {lin }}$ and a corresponding Green operator $\mathcal{G}_{\text {lin }}$. Application of the parametrix $\mathcal{P}_{\text {lin }}$ to HF equations at successive iterations yields the following equation

$$
\begin{aligned}
\phi_{i}^{(n)}-\phi_{i}^{(m)} & =P_{\operatorname{lin}}\left[\left(V^{(m-1)}-V^{(n-1)}\right) \phi_{i}^{(m)}-V^{(n-1)}\left(\phi_{i}^{(n)}-\phi_{i}^{(m)}\right)\right] \\
& +\left(\varepsilon_{i}^{(n)}-\varepsilon_{i}^{(m)}\right) P_{\operatorname{lin}} \phi_{i}^{(n)}+\left(\varepsilon_{i}^{(m)}-\varepsilon\right) P_{\operatorname{lin}}\left(\phi_{i}^{(n)}-\phi_{i}^{(m)}\right) \\
& -\mathcal{G}_{\operatorname{lin}}\left(\phi_{i}^{(n)}-\phi_{i}^{(m)}\right) .
\end{aligned}
$$

For the bootstrap argument we consider again a sequence of weighted Sobolev spaces $\mathcal{K}_{P_{k}}^{k, \gamma}\left(X^{\wedge}\right)$, $k=1,2, \ldots$, with weight data $(\gamma,(\gamma-k-1,0])$. Let us assume that the eigenfunctions $\phi_{i}^{(n)}$ form a Cauchy sequence i.e.

$$
\lim _{n, m \rightarrow \infty}\left\|\phi_{i}^{(n)}-\phi_{i}^{(m)}\right\|_{\mathcal{K}_{P_{k}}^{k, \gamma}\left(X^{\wedge}\right)}=0
$$

and

$$
\lim _{n, m \rightarrow \infty}\left\|\left(V^{(m-1)}-V^{(n-1)}\right) \phi_{i}^{(m)}-V^{(n-1)}\left(\phi_{i}^{(n)}-\phi_{i}^{(m)}\right)\right\|_{\mathcal{K}_{P_{k}}^{k, \gamma}\left(X^{\wedge}\right)}=0 .
$$

Given $\lim _{n, m \rightarrow \infty}\left|\varepsilon_{i}^{(n)}-\varepsilon_{i}^{(m)}\right|=0$, it follows immediately from (2.3) and (2.27) that

$$
\lim _{n, m \rightarrow \infty}\left\|\phi_{i}^{(n)}-\phi_{i}^{(m)}\right\|_{\mathcal{K}_{P_{k+2}}^{k+2, \gamma}\left(X^{\wedge}\right)}=0 .
$$

The bootstrap argument provides a series of Cauchy sequences

$$
\cdots \Longrightarrow \lim _{n, m \rightarrow \infty}\left\|\phi_{i}^{(n)}-\phi_{i}^{(m)}\right\|_{\mathcal{K}_{P_{k}}^{k, \gamma}\left(X^{\wedge}\right)}=0 \Longrightarrow \lim _{n, m \rightarrow \infty}\left\|\phi_{i}^{(n)}-\phi_{i}^{(m)}\right\|_{\mathcal{K}_{P_{k+2}}^{k+2, \gamma}\left(X^{\wedge}\right)}=0 \Longrightarrow \cdots
$$

where convergence of a Cauchy sequence $\phi_{i}^{(n)}$ in the Frechet space $\mathcal{K}_{P_{k}}^{k, \gamma}\left(X^{\wedge}\right)$ implies strong convergence s- $\lim _{n \rightarrow \infty} \phi_{i}^{(n)}=\phi_{i} \in \mathcal{K}_{P_{k}}^{k, \gamma}\left(X^{\wedge}\right)$. 
It has been proven by Cancès and Le Bris, cf. Ref. [2], that the level-shifting algorithm (2.1) generates a sequence of eigenvalues and eigenfunctions from which a subsequence $\varepsilon_{i}^{(n)}, \phi_{i}^{(n)}$, can be extracted that converges in $\mathbb{R}$ and $H^{1}\left(\mathbb{R}^{3}\right)$, respectively. Furthermore, the subsequence $\phi_{i}^{(n-1)}$ converges in $H^{1}\left(\mathbb{R}^{3}\right)$ to the same limit, cf. our discussion of the level-shifting algorithm in Section 2. Our starting point is $\mathcal{K}^{0,0}\left(X^{\wedge}\right)$ which is equivalent to $L^{2}\left(\mathbb{R}^{3}\right)$. We consider the two terms in the norm (2.28) separately. The second term is given explicitly by

$$
\begin{aligned}
& V^{(n-1)}\left(\phi_{i}^{(n)}-\phi_{i}^{(m)}\right)=\sum_{j=1}^{N / 2}\left[\left(\phi_{i}^{(n)}-\phi_{i}^{(m)}\right) \frac{2}{|x|} *\left|\phi_{j}^{(n-1)}\right|^{2}\right. \\
& \left.\quad-\phi_{j}^{(n-1)} \frac{1}{|x|} *\left(\phi_{j}^{(n-1)}\left(\phi_{i}^{(n)}-\phi_{i}^{(m)}\right)\right)-b \phi_{j}^{(n-1)}\left\langle\phi_{i}^{(n)}-\phi_{i}^{(m)}, \phi_{j}^{(n-1)}\right\rangle_{L^{2}\left(\mathbb{R}^{3}\right)}\right] .
\end{aligned}
$$

From (2.12), we obtain the following estimates for the individual terms

$$
\begin{gathered}
\left\|\left(\phi_{i}^{(n)}-\phi_{i}^{(m)}\right) \frac{2}{|x|} *\left|\phi_{j}^{(n-1)}\right|^{2}\right\|_{L^{2}\left(\mathbb{R}^{3}\right)} \lesssim\left\|\phi_{j}^{(n-1)}\right\|_{H^{1}\left(\mathbb{R}^{3}\right)}\left\|\phi_{j}^{(n-1)}\right\|_{L^{2}\left(\mathbb{R}^{3}\right)}\left\|\phi_{i}^{(n)}-\phi_{i}^{(m)}\right\|_{L^{2}\left(\mathbb{R}^{3}\right)} \\
\left\|\phi_{j}^{(n-1)} \frac{1}{|x|} *\left(\phi_{j}^{(n-1)}\left(\phi_{i}^{(n)}-\phi_{i}^{(m)}\right)\right)\right\|_{L^{2}\left(\mathbb{R}^{3}\right)} \lesssim\left\|\phi_{j}^{(n-1)}\right\|_{H^{1}\left(\mathbb{R}^{3}\right)}\left\|\phi_{j}^{(n-1)}\right\|_{L^{2}\left(\mathbb{R}^{3}\right)}\left\|\phi_{i}^{(n)}-\phi_{i}^{(m)}\right\|_{L^{2}\left(\mathbb{R}^{3}\right)} .
\end{gathered}
$$

A corresponding estimate for the projection term follows immediately from Schwarz's inequality. Similar arguments apply to the first term given by

$$
\begin{aligned}
& \left(V^{(m-1)}-V^{(n-1)}\right) \phi_{i}^{(m)}=\sum_{j=1}^{N / 2}\left[\phi_{i}^{(m)} \frac{2}{|x|} *\left(\left|\phi_{j}^{(m-1)}\right|^{2}-\left|\phi_{j}^{(n-1)}\right|^{2}\right)\right. \\
& \quad-\phi_{j}^{(m-1)} \frac{1}{|x|} *\left(\left(\phi_{j}^{(m-1)}-\phi_{j}^{(n-1)}\right) \phi_{i}^{(m)}\right)+\left(\phi_{j}^{(n-1)}-\phi_{j}^{(m-1)}\right) \frac{1}{|x|} *\left(\phi_{j}^{(n-1)} \phi_{i}^{(m)}\right) \\
& \left.\quad-b\left(\phi_{j}^{(m-1)}-\phi_{j}^{(n-1)}\right)\left\langle\phi_{i}^{(m)}, \phi_{j}^{(m-1)}\right\rangle_{L^{2}\left(\mathbb{R}^{3}\right)}+b \phi_{j}^{(n-1)}\left\langle\phi_{i}^{(m)}, \phi_{j}^{(n-1)}-\phi_{j}^{(m-1)}\right\rangle_{L^{2}\left(\mathbb{R}^{3}\right)}\right] .
\end{aligned}
$$

Again, from (2.12), we get estimates for the individual terms

$$
\begin{aligned}
& \left\|\phi_{i}^{(m)} \frac{2}{|x|} *\left(\left|\phi_{j}^{(m-1)}\right|^{2}-\left|\phi_{j}^{(n-1)}\right|^{2}\right)\right\|_{L^{2}\left(\mathbb{R}^{3}\right)} \lesssim\left\|\phi_{i}^{(m)}\right\|_{L^{2}\left(\mathbb{R}^{3}\right)}\left\|\phi_{j}^{(m-1)}-\phi_{j}^{(n-1)}\right\|_{L^{2}\left(\mathbb{R}^{3}\right)} \\
& \times\left(\left\|\phi_{j}^{(m-1)}\right\|_{H^{1}\left(\mathbb{R}^{3}\right)}+\left\|\phi_{j}^{(n-1)}\right\|_{\left.H^{1} \mathbb{R}^{3}\right)}\right), \\
& \left\|\phi_{j}^{(m-1)} \frac{1}{|x|} *\left(\left(\phi_{j}^{(m-1)}-\phi_{j}^{(n-1)}\right) \phi_{i}^{(m)}\right)\right\|_{L^{2}\left(\mathbb{R}^{3}\right)} \lesssim\left\|\phi_{j}^{(m-1)}\right\|_{L^{2}\left(\mathbb{R}^{3}\right)}\left\|\phi_{i}^{(m)}\right\|_{H^{1}\left(\mathbb{R}^{3}\right)}\left\|\phi_{j}^{(m-1)}-\phi_{j}^{(n-1)}\right\|_{L^{2}\left(\mathbb{R}^{3}\right)}, \\
& \left\|\left(\phi_{j}^{(n-1)}-\phi_{j}^{(m-1)}\right) \frac{1}{|x|} *\left(\phi_{j}^{(n-1)} \phi_{i}^{(m)}\right)\right\|_{L^{2}\left(\mathbb{R}^{3}\right)} \lesssim\left\|\phi_{j}^{(n-1)}\right\|_{L^{2}\left(\mathbb{R}^{3}\right)}\left\|\phi_{i}^{(m)}\right\|_{H^{1}\left(\mathbb{R}^{3}\right)}\left\|\phi_{j}^{(m-1)}-\phi_{j}^{(n-1)}\right\|_{L^{2}\left(\mathbb{R}^{3}\right)} .
\end{aligned}
$$

These estimates together with (2.27) imply s- $\lim _{n \rightarrow \infty} \phi_{i}^{(n)}=\phi_{i} \in \mathcal{K}_{P_{1}}^{1, \gamma}\left(X^{\wedge}\right)$ for $\gamma<3 / 2$. Actually it implies slightly more with respect to the Sobolev regularity. The reason for this downgrading will become clear in the following.

The finite dimensional spaces $\mathcal{E}_{P_{k}}^{\gamma}$ are isomorphic to $\mathbb{R}^{m}$ for a $m \in \mathbb{N}$, we can therefore reduce convergence considerations to individual sequences of coefficients $\left\{c_{j}^{(n)}\right\}$ where the following remark turns out to be helpful. 
Remark 2. Given two Cauchy sequences $\left\{c_{j}^{(n)}\right\},\left\{c_{k}^{(n)}\right\}$ in $\mathbb{R}$. Then the product $\left\{c_{j}^{(n)} c_{k}^{(n)}\right\}$ is a Cauchy sequence again.

Proof.

$$
\begin{aligned}
\left|c_{j}^{(n)} c_{k}^{(n)}-c_{j}^{(m)} c_{k}^{(m)}\right| & =\left|c_{j}^{(n)}\left(c_{k}^{(n)}-c_{k}^{(m)}\right)-\left(c_{j}^{(m)}-c_{j}^{(n)}\right) c_{k}^{(m)}\right| \\
& \leq\left|c_{j}^{(n)}\right|\left|c_{k}^{(n)}-c_{k}^{(m)}\right|+\left|c_{j}^{(n)}-c_{j}^{(m)}\right|\left|c_{k}^{(m)}\right|
\end{aligned}
$$

Next, we consider products of Cauchy sequences in weighted Sobolev spaces $\mathcal{K}^{s, \gamma}$.

Proposition 3. The functions in $\mathcal{K}^{s, \gamma}$, with $s \in \mathbb{Z}_{+}, s>2$ and $\gamma \geq s$, constitute an algebra. Given two Cauchy sequences $\left\{f_{n}\right\},\left\{g_{n}\right\} \in \mathcal{K}^{s, \gamma}$ in such function spaces, then the product $\left\{f_{n} g_{n}\right\} \in \mathcal{K}^{s, \gamma}$ is a Cauchy sequence again.

Proof. According to definition (1.10), the proof can be subdived in two parts. First we consider $\omega\left(f_{n} g_{n}-f_{m} g_{m}\right)$ in the weighted Sobolev space $\mathcal{H}^{s, \gamma}\left(X^{\wedge}\right)$. For convenience, we introduce another cut-off function $\tilde{\omega}$ with $\tilde{\omega}=1$ on $\operatorname{supp} \omega$ and $\operatorname{supp} \tilde{\omega}$ contained in a bounded domain $\Omega$. Let us define $\tilde{f}_{n}:=\omega f_{n}$ and $\tilde{g}_{n}:=\tilde{\omega} g_{n}$. With this we get the following estimate

$$
\begin{aligned}
\left\|\omega\left(f_{n} g_{n}-f_{m} g_{m}\right)\right\|_{\mathcal{H}^{s, \gamma}\left(X^{\wedge}\right)} & =\left\|\tilde{f}_{n} \tilde{g}_{n}-\tilde{f}_{m} \tilde{g}_{m}\right\|_{\mathcal{H}^{s, \gamma}\left(X^{\wedge}\right)} \\
& =\sum_{|\alpha| \leq s}\left\||x|^{-\gamma+|\alpha|} \partial^{\alpha}\left(\tilde{f}_{n} \tilde{g}_{n}-\tilde{f}_{m} \tilde{g}_{m}\right)\right\|_{L^{2}(\Omega)} \\
& \lesssim \sum_{|\alpha| \leq s} \sum_{\alpha_{1}+\alpha_{2}=\alpha}\left[\left\||x|^{-\gamma+|\alpha|} \partial^{\alpha_{1}} \tilde{f}_{n}\left(\partial^{\alpha_{2}} \tilde{g}_{n}-\partial^{\alpha_{2}} \tilde{g}_{m}\right)\right\|_{L^{2}(\Omega)}\right. \\
& \left.+\left\||x|^{-\gamma+|\alpha|}\left(\partial^{\alpha_{1}} \tilde{f}_{n}-\partial^{\alpha_{1}} \tilde{f}_{m}\right) \partial^{\alpha_{2}} \tilde{g}_{m}\right\|_{L^{2}(\Omega)}\right] .
\end{aligned}
$$

For the next estimate let us assume w.l.o.g. that $\left|\alpha_{1}\right| \leq\left|\alpha_{2}\right|$ and therefore $\left|\alpha_{1}\right| \leq\lfloor s / 2\rfloor$. Furthermore we require the Sobolev imbedding $H^{s}(\Omega) \hookrightarrow C_{B}^{l}(\Omega):=\left\{u \in C^{l}(\Omega): \partial^{\alpha} u \in L^{\infty}(\Omega)\right.$ for $\left.|\alpha| \leq l\right\}$ for $0 \leq l<s-3 / 2$ (cf. Ref. [5]). With this we obtain for the individual terms

$$
\begin{aligned}
&\left\||x|^{-\gamma+|\alpha|} \partial^{\alpha_{1}} \tilde{f}_{n}\left(\partial^{\alpha_{2}} \tilde{g}_{n}-\partial^{\alpha_{2}} \tilde{g}_{m}\right)\right\|_{L^{2}(\Omega)} \leq\left\||x|^{\left|\alpha_{1}\right|} \partial^{\alpha_{1}} \tilde{f}_{n}\right\|_{L^{\infty}(\Omega)}\left\||x|^{-\gamma+\left|\alpha_{2}\right|}\left(\partial^{\alpha_{2}} \tilde{g}_{n}-\partial^{\alpha_{2}} \tilde{g}_{m}\right)\right\|_{L^{2}(\Omega)} \\
& \lesssim\left\|\tilde{f}_{n}\right\|_{H^{s}(\Omega)}\left\|\tilde{g}_{n}-\tilde{g}_{m}\right\|_{\mathcal{H}^{s, \gamma}\left(X^{\wedge}\right)} \\
& \lesssim\left\|\tilde{f}_{n}\right\|_{\mathcal{H}^{s, \gamma}\left(X^{\wedge}\right)}\left\|\tilde{g}_{n}-\tilde{g}_{m}\right\|_{\mathcal{H}^{s, \gamma}\left(X^{\wedge}\right)}, \\
&\left\||x|^{-\gamma+|\alpha|}\left(\partial^{\alpha_{1}} \tilde{f}_{n}-\partial^{\alpha_{1}} \tilde{f}_{m}\right) \partial^{\alpha_{2}} \tilde{g}_{m}\right\|_{L^{2}(\Omega)} \lesssim\left\|\tilde{f}_{n}-\tilde{f}_{m}\right\|_{\mathcal{H}^{s, \gamma}\left(X^{\wedge}\right)}\left\|\tilde{g}_{m}\right\|_{\mathcal{H}^{s, \gamma}\left(X^{\wedge}\right)} .
\end{aligned}
$$

The remaining part $(1-\omega)\left(f_{n} g_{n}-f_{m} g_{m}\right)$, in the space $H^{s}\left(\mathbb{R}^{3}\right)$, can be dealt with similar arguments.

Together with Remark (2), the previous proposition can be used to prove the following lemma.

Lemma 5. The functions in $\mathcal{K}_{P_{k}}^{k, \gamma}\left(X^{\wedge}\right)$, with $k>2$ and $\gamma<3 / 2$, constitute an algebra. Given two Cauchy sequences $\left\{f_{n}\right\},\left\{g_{n}\right\}$ in such function spaces, then the product $\left\{f_{n} g_{n}\right\} \in \mathcal{K}_{P_{k}}^{k, \gamma}\left(X^{\wedge}\right)$ is a Cauchy sequence again. 
Proof. We decompose the functions according to $f_{n}=v_{n}+w_{n}, g_{n}=\tilde{v}_{n}+\tilde{w}_{n}$ with $v_{n}, \tilde{v}_{n} \in \mathcal{E}_{P_{k}}^{\gamma}\left(X^{\wedge}\right)$ and $w_{n}, \tilde{w}_{n} \in \mathcal{K}_{\Theta_{k}}^{k, \gamma}\left(X^{\wedge}\right)$. With this, the products splits into

$$
f_{n} g_{n}=v_{n} \tilde{v}_{n}+v_{n} \tilde{w}_{n}+w_{n} \tilde{v}_{n}+w_{n} \tilde{w}_{n}
$$

The proposition follows immediately for the first and fourth term on the right hand side from Remark 2 and Proposition 3, respectively. The mixed terms $v_{n} \tilde{w}_{n}$ and $w_{n} \tilde{v}_{n}$ belong to $\mathcal{K}_{\Theta_{k}}^{k, \gamma}\left(X^{\wedge}\right)$ and can be handled like in the proof of Proposition 3 where we utilize that $\left\{|x|^{\alpha_{1}} \partial^{\alpha_{1}} v_{n}\right\}$ are Cauchy sequences in $L^{\infty}(\Omega)$.

The following corollary is an immediate consequence of the proof of Proposition 3 and Lemma 5 .

Corollary 1. Given a bounded sequence $\left\{f_{n}\right\}$ and a Cauchy sequence $\left\{g_{n}\right\}$ in $\mathcal{K}_{P_{k}}^{k, \gamma}\left(X^{\wedge}\right)$. Then

$$
\lim _{n, m \rightarrow \infty}\left\|f_{l}\left(g_{n}-g_{m}\right)\right\|_{\mathcal{K}_{P_{k}}^{k, \gamma}\left(X^{\wedge}\right)}=0
$$

uniformly in $l$.

We turn now to estimates for the nonlinear quantities (2.29) and (2.30) in the weighted Sobolev space with asymptotics $\mathcal{K}_{P_{k}}^{k, \gamma}\left(X^{\wedge}\right)$. The estimates are given for $k>2$ and a subsequent argument shows how to bridge a remaining gap in the bootstrap argument. Let us start with the first term on the right hand side of (2.29). Our discussion follows here almost literally the preceding proof of Theorem 1. For the convenience of the reader, we repeat the basic arguments since we are now concerned with Cauchy sequences instead of single function estimates. We decompose $\phi_{i}^{(n)}=$ $v_{i}^{(n)}+w_{i}^{(n)}, \phi_{i}^{(m)}=v_{i}^{(m)}+w_{i}^{(m)}$ and $\phi_{j}^{(n-1)}=v_{j}^{(n-1)}+w_{j}^{(n-1)}$ into its components in $\mathcal{E}_{P_{k}}^{\gamma}\left(X^{\wedge}\right)$ and $\mathcal{K}_{\Theta_{k}}^{k, \gamma}\left(X^{\wedge}\right)$. Correspondingly, we decompose the quadratic term

$$
\left|\phi_{j}^{(n-1)}\right|^{2}=v_{q}^{(n-1)}+w_{q}^{(n-1)}
$$

with

$$
v_{q}^{(n-1)}:=\left(v_{j}^{(n-1)}\right)^{2} \in \mathcal{S}_{P_{\infty}}^{\gamma}\left(X^{\wedge}\right), \text { and } w_{q}^{(n-1)}:=2 v_{j}^{(n-1)} w_{j}^{(n-1)}+\left(w_{j}^{(n-1)}\right)^{2} \in \mathcal{K}_{\Theta_{k}}^{k, \gamma}\left(X^{\wedge}\right) .
$$

Then it follows from Lemmas 1 and 2 that

$$
\lim _{n, m \rightarrow \infty}\left\|\left(v_{i}^{(n)}-v_{i}^{(m)}\right) \frac{1}{|x|} * v_{q}^{(n-1)}\right\|_{\mathcal{K}_{P_{k}}^{k, \gamma}\left(X^{\wedge}\right)}=0 .
$$

For the second combination

$$
\left(w_{i}^{(n)}-w_{i}^{(m)}\right) \frac{1}{|x|} * v_{q}^{(n-1)} \in \mathcal{K}_{\Theta_{k}}^{k, \gamma}\left(X^{\wedge}\right),
$$

we introduce a cut-off function $\eta$ with supp $\eta \subset \Omega$ and consider first the spaces $\mathcal{H}^{k, k+1-\epsilon}\left(X^{\wedge}\right)$ for $\epsilon>0$ where we get

$$
\begin{aligned}
\| \eta\left(w_{i}^{(n)}-\right. & \left.w_{i}^{(m)}\right) \frac{1}{|x|} * v_{q}^{(n-1)}\left\|_{\mathcal{H}^{k, k+1-\epsilon}\left(X^{\wedge}\right)} \lesssim \sum_{|\alpha| \leq k} \sum_{\alpha_{1}+\alpha_{2}=\alpha}\right\||x|^{\left|\alpha_{1}\right|} \partial^{\alpha_{1}} \frac{1}{|x|} * v_{q}^{(n-1)} \|_{L^{\infty}(\Omega)} \\
& \times\left\||x|^{-k-1+\epsilon+\left|\alpha_{2}\right|} \partial^{\alpha_{2}}\left[\eta\left(w_{i}^{(n)}-w_{i}^{(m)}\right)\right]\right\|_{L^{2}(\Omega)} \lesssim\left\|\eta\left(w_{i}^{(n)}-w_{i}^{(m)}\right)\right\|_{\mathcal{H}^{k, k+1-\epsilon}\left(X^{\wedge}\right)} .
\end{aligned}
$$


The uniform boundedness of the factors in $L^{\infty}$ norm is a consequence of Lemma 1. Similar estimates can be obtained for the $(1-\eta)(\cdot)$ term in $H^{k}\left(\mathbb{R}^{3}\right)$. The third combination

$$
\left(w_{i}^{(n)}-w_{i}^{(m)}\right) \frac{1}{|x|} * w_{j}^{(n-1)} \in \mathcal{K}_{\Theta_{k}}^{k, \gamma}\left(X^{\wedge}\right),
$$

can be handled likewise, except that the $L^{\infty}$ terms again require particular attention. Like in the proof of Theorem 1, we first decompose the Coulomb potential into $L^{2}\left(\mathbb{R}^{3}\right)+L^{\infty}\left(\mathbb{R}^{3}\right)$ according to (2.13). According to (2.14), we obtain, by applying Young's inequality to the Yukawa part of the potential, the estimate

$$
\left\|\mathcal{Y} * \partial^{\alpha_{1}} w_{q}^{(n-1)}\right\|_{L^{\infty}(\Omega)} \lesssim\left\|w_{q}^{(n-1)}\right\|_{\mathcal{K}^{k, k+1-\epsilon}\left(X^{\wedge}\right)} .
$$

The bounded part of the Coulomb can be estimated, according to (2.15), using the other variant of Young's inequality

$$
\left\|\mathcal{W} * \partial^{\alpha_{1}} w_{q}^{(n-1)}\right\|_{L^{\infty}(\Omega)} \lesssim\left\|v_{j}^{(n-1)}\right\|_{\mathcal{K}_{P_{k}}^{k, \gamma}\left(X^{\wedge}\right)}\left\|w_{j}^{(n-1)}\right\|_{\mathcal{K}^{k, k+1-\epsilon}\left(X^{\wedge}\right)}+\left\|w_{j}^{(n-1)}\right\|_{\mathcal{K}^{k, k+1-\epsilon}\left(X^{\wedge}\right)}^{2} .
$$

It remains to consider the fourth combination

$$
\left(v_{i}^{(n)}-v_{i}^{(m)}\right) \frac{1}{|x|} * w_{q}^{(n-1)},
$$

which restricts to a bounded domain $\Omega$ because of the compact support of the functions $v_{i}^{(n)}$. Again, we perform a Taylor series decomposition of the convolution term

$$
\frac{1}{|x|} * w_{q}^{(n-1)}=\sum_{|\beta| \leq k-1} c_{\beta}^{(n-1)} x^{\beta}+W_{k}^{(n-1)}, \text { with } c_{\beta}^{(n-1)}:=\frac{1}{\beta !} \int \frac{\partial^{\beta} w_{q}^{(n-1)}(y)}{|y|} d y
$$

with formal residuum

$$
W_{k}^{(n-1)}:=\frac{1}{|x|} * w_{q}^{(n-1)}-\sum_{|\beta| \leq k-1} c_{\beta}^{(n-1)} x^{\beta} .
$$

The Taylor expansion converges as a Cauchy sequence

$$
\lim _{n, m \rightarrow \infty}\left\|\left(v_{i}^{(n)}-v_{i}^{(m)}\right) \sum_{|\beta| \leq k-1} c_{\beta}^{(n-1)} x^{\beta}\right\|_{\mathcal{K}_{P_{k}}^{k, \gamma}\left(X^{\wedge}\right)}=0
$$

because the constants $c_{\beta}^{(n-1)}$ are bounded according to the estimates (2.31) and (2.32). Following (2.17), the derivatives of the residuum $W_{k}^{(n-1)}$ can be estimated

$|x|^{|\alpha|-k}\left|\partial^{\alpha} W_{k}^{(n-1)}\right| \lesssim\left\|w_{q}^{(n-1)}\right\|_{\mathcal{K}^{k, k+1-\epsilon}\left(X^{\wedge}\right)}+\left\|v_{j}^{(n-1)}\right\|_{\mathcal{K}_{P_{k}}^{k, \gamma}\left(X^{\wedge}\right)}\left\|w_{j}^{(n-1)}\right\|_{\mathcal{K}^{k, k+1-\epsilon}\left(X^{\wedge}\right)}+\left\|w_{j}^{(n-1)}\right\|_{\mathcal{K}^{k, k+1-\epsilon}\left(X^{\wedge}\right)}^{2}$,

using an explicit expression for the remainder of the Taylor expansion together with (2.31) and (2.32). It is sufficient to consider for the residuum the space $\mathcal{H}^{k, k+1-\epsilon}\left(X^{\wedge}\right)$ where an estimate

$$
\left\|\left(v_{i}^{(n)}-v_{i}^{(m)}\right) W_{k}^{(n-1)}\right\|_{\mathcal{H}^{k, k+1-\epsilon}\left(X^{\wedge}\right)} \lesssim\left\|v_{i}^{(n)}-v_{i}^{(m)}\right\|_{\mathcal{K}_{P_{k}}^{k, \gamma}\left(X^{\wedge}\right)} \sum_{|\alpha| \leq k}\left\||x|^{|\alpha|-k} \partial^{\alpha} W_{k}^{(n-1)}\right\|_{L^{\infty}(\Omega)},
$$

analogous to (2.18), demonstrates convergence of the Cauchy sequence. 
The second term on the right hand side of (2.29) can be treated along the lines of the previous one. Again, we decompose the convolution term

$$
\begin{gathered}
\phi_{j}^{(n-1)}\left(\phi_{i}^{(n)}-\phi_{i}^{(m)}\right)=v^{(n, m)}+w^{(n, m)} \text { with } v^{(n, m)}:=v_{j}^{(n-1)}\left(v_{i}^{(n)}-v_{i}^{(m)}\right) \in \mathcal{S}_{P_{\infty}}^{\gamma}\left(X^{\wedge}\right) \\
\text { and } w^{(n, m)}:=v_{j}^{(n-1)}\left(w_{i}^{(n)}-w_{i}^{(m)}\right)+w_{j}^{(n-1)}\left(v_{i}^{(n)}-v_{i}^{(m)}\right)+w_{j}^{(n-1)}\left(w_{i}^{(n)}-w_{i}^{(m)}\right) \in \mathcal{K}_{\Theta_{k}}^{k, \gamma}\left(X^{\wedge}\right) .
\end{gathered}
$$

Both parts have separate limits

$$
\lim _{n, m \rightarrow \infty}\left\|v^{(n, m)}\right\|_{\mathcal{K}_{P_{k}}^{k, \gamma}\left(X^{\wedge}\right)}=0, \text { and } \lim _{n, m \rightarrow \infty}\left\|w^{(n, m)}\right\|_{\mathcal{K}_{\Theta_{k}}^{k, \gamma}\left(X^{\wedge}\right)}=0
$$

which ensures that the right hand side of the estimate (2.31) vanish for $n, m \rightarrow \infty$. Finally we just want to mention that the first term on the right hand side of (2.30) reduces to the previous case using the identity

$$
\left(\phi_{j}^{(m-1)}\right)^{2}-\left(\phi_{j}^{(n-1)}\right)^{2}=\left(\phi_{j}^{(m-1)}-\phi_{j}^{(n-1)}\right) \phi_{j}^{(m-1)}+\left(\phi_{j}^{(m-1)}-\phi_{j}^{(n-1)}\right) \phi_{j}^{(n-1)} .
$$

All remaining terms in (2.30) are similar to the terms discussed explicitly.

Altogether, this proves the limit $(2.28)$ for $\mathcal{K}_{P_{k}}^{k, \gamma}\left(X^{\wedge}\right)$ with $k>2$. It remains to consider the case $k=1$ which has so far been skipped in our discussion. Going through the proofs of Proposition 3 and Lemma 5 it can be seen that these proofs go through even in the case $k=1$ using the additional regularity from the fact that $\mathcal{P}_{\text {lin }}$ provides a continous map from $\mathcal{K}^{0,0}\left(X^{\wedge}\right)$ into $\mathcal{K}^{2,2}\left(X^{\wedge}\right)$. This is sufficient for the remaining steps required for the proof of the limit $(2.28)$ for $\mathcal{K}_{P_{1}}^{1, \gamma}\left(X^{\wedge}\right)$.

So far we have shown that the iterative solutions $\phi_{i}^{(n)}$ of the level-shifting algorithm converge to solutions $\phi_{i}$ of the HF equation (1.3) in the weighted Sobolev space with asymptotics $\mathcal{K}_{P_{\infty}^{\infty}}^{\infty, \gamma}\left(X^{\wedge}\right)$ i.e.

$$
\lim _{n \rightarrow \infty}\left\|\phi_{i}^{(n)}-\phi_{i}\right\|_{\mathcal{K}_{P_{\infty}}^{\infty, \gamma}\left(X^{\wedge}\right)}=0 \text { for all } i=1, N / 2 \text {. }
$$

What remains to show is $\phi_{i} \in \mathcal{S}_{P_{\infty}}^{\gamma}\left(X^{\wedge}\right)$, which finishes the proof that the solutions are asymptotically well behaved. The proof can be simplified using the trick of Lieb and Simon [8] to represent the nonlocal HF equations as a system of coupled local equations. For this they introduced the operator matrix $\mathbf{h}$ with entries

$$
h_{i, j}:=\delta_{i, j}\left(\mathfrak{h}_{\text {lin }}+V_{H}-\varepsilon_{i}\right)+\mathcal{U}_{i, j}, \quad \text { with } \mathcal{U}_{i, j}:=-\int \frac{\phi_{i}(y) \phi_{j}(y)}{|x-y|} d y,
$$

by which the HF equations can be written in the form $\mathbf{h} \phi=0$. It will be shown in the following that the operator matrix $\mathbf{h}$ actually corresponds to an elliptic operator. This means that a parametrix $\mathbf{P}$ and a Green operator $\mathbf{G}$ exists from which we obtain the desired result

$$
\mathbf{P h} \phi=\phi+\mathbf{G} \phi=0 \longrightarrow \phi_{i} \in \mathcal{S}_{P_{\infty}}^{\gamma}
$$

It is evident from the properties of the Sobolev space $\mathcal{K}_{P_{\infty}}^{\infty, \gamma}$ that the short-range behaviour of the nonlinear potentials in the operator matrix (2.33) is in correspondence with the ellipticity requirement mentioned in Section 3.2 and we are left with the estimates for the long-range behaviour (cf. (2.8)),

$$
\left|\partial^{\alpha}\left[(1-\omega) \frac{1}{|x|} *\left(\tilde{\phi}_{i} \tilde{\phi}_{j}\right)\right]\right| \lesssim(1+|x|)^{-1-|\alpha|}, \quad \text { with } \tilde{\phi}_{i}=(1-\omega) \phi_{i} .
$$

The following propositions enable us to get control on the long-range behaviour of convolutions with the Coulomb potential. 
Proposition 4. The convolution of a function $u \in \mathcal{K}_{P}^{s, \gamma}\left(X^{\wedge}\right)$, with the Coulomb potential can be expressed through

$$
\begin{gathered}
\frac{1}{|x|} * u=-\mathcal{P}_{m} u+(-1)^{m} c^{m} \frac{1}{|x|} * \mathcal{P}_{c}^{m} u-\frac{1}{|x|} * \mathcal{G}_{m} u \\
\text { with } \mathcal{P}_{m}:=4 \pi \mathcal{P}_{c} \sum_{i=0}^{m-1}(-1)^{i} c^{i} \mathcal{P}_{c}^{i}, \quad \text { and } \mathcal{G}_{m}:=\mathcal{G}_{c} \sum_{i=0}^{m-1}(-1)^{i} c^{i} \mathcal{P}_{c}^{i}
\end{gathered}
$$

where $\mathcal{P}_{c}$ and $\mathcal{G}_{c}$ are the parametrix and Green operator of a shifted Laplacian for weight data $\underline{g}=(\gamma, \gamma-2, \Theta)$ with $3 / 2-\gamma \in \mathbb{R} \backslash \mathbb{Z}, \gamma<3 / 2$ and $\Theta=(-\infty, 0]$, respectively, i.e.

$$
(\Delta-c) \mathcal{P}_{c}=I+\mathcal{G}_{c} \text { with } c>0
$$

Proof. The existence of a parametrix for a shifted Laplacian $\Delta-c$ with $c>0$ is an immediate consequence of the general theory of elliptic operators, cf. Theorem 8, p. 259 of Ref. [3]. Recursive application of (2.35) yields

$$
\begin{aligned}
u & =\Delta \mathcal{P}_{c} u-c \mathcal{P}_{c} u-\mathcal{G}_{c} u \\
& =\Delta \mathcal{P}_{c} u-c \Delta \mathcal{P}_{c}^{2} u+c^{2} \mathcal{P}_{c}^{2} u+c \mathcal{G}_{c} \mathcal{P}_{c} u-\mathcal{G}_{c} u \\
& \vdots \\
& =\Delta \mathcal{P}_{c} \sum_{i=0}^{m-1}(-1)^{i} c^{i} \mathcal{P}_{c}^{i} u+(-1)^{m} c^{m} \mathcal{P}_{c}^{m} u-\mathcal{G}_{c} \sum_{i=0}^{m-1}(-1)^{i} c^{i} \mathcal{P}_{c}^{i} u .
\end{aligned}
$$

This Poisson type equation has a unique solution (2.34) obtained through convolution with the Coulomb potential. Uniqueness follows from the fact that $\mathcal{P}_{m} u \in L^{2}\left(\mathbb{R}^{3}\right)$ and therefore belongs to $\mathcal{S}^{\prime}\left(\mathbb{R}^{3}\right)$. In $\mathcal{S}^{\prime}\left(\mathbb{R}^{3}\right)$ the only distributional solutions of $\Delta w=0$ are harmonic polynomials, cf. Ref. [12] p. 147 , which do not belong to $L^{2}\left(\mathbb{R}^{3}\right)$.

Proposition 5. Given $\mathcal{A} \in C^{n}\left(X^{\wedge}, \underline{g}\right)$ with $n<-s-\frac{3}{2}$ and $s \in \mathbb{Z}_{+}$. For any $u \in \mathcal{K}_{P}^{s, \gamma}\left(X^{\wedge}\right)$, which in addition satisfies $\sup _{x}\left|x^{\beta} u(x)\right|<\infty$ for all $|\beta| \geq 0$, and arbitrary cut-off function $\omega$, the following asymptotic estimate can be derived

$$
\left|\partial_{x}^{\alpha} \mathcal{A}(1-\omega) u\right| \lesssim\left(1+|x|^{2}\right)^{-m}, \text { for all }|\alpha| \leq s \text { and } m \in \mathbb{Z}_{+}
$$

Proof. First we perform a canonical decomposition of $\mathcal{A}$ (cf. Ref. [3])

$$
\mathcal{A}=\mathcal{A}^{\prime}+\tilde{\mathcal{A}}+\tilde{\mathcal{G}} \text { with } \mathcal{A}^{\prime}:=\eta \mathcal{A}_{0} \tilde{\eta} \text { and } \tilde{\mathcal{A}}:=(1-\eta) \mathcal{A}_{1}(1-\tilde{\tilde{\eta}})
$$

for appropriately chosen cut-off functions $\tilde{\eta} \equiv 1$ on $\operatorname{supp} \eta, \eta \equiv 1$ on $\operatorname{supp} \tilde{\tilde{\eta}}$ and $\omega \tilde{\eta}=\tilde{\eta}$, where $\mathcal{A}_{1}$ corresponds to a pseudo-differential operator on $\mathbb{R}^{3}$ with symbol $a(x, \xi)$ in the symbol class $S^{n, 0}\left(\mathbb{R}^{3} \times \mathbb{R}^{3}\right)$. It is obvious from (2.36) that it is sufficient to consider $\mathcal{A}_{1}$, where we adapt a standard proof that pseudo-differential operators on $\mathbb{R}^{3}$ map $\mathcal{S}$ into $\mathcal{S}$, cf. p. 232ff in Ref. [11]. Following the proof in Ref. [11], we introduce the operator

$$
L_{\xi}:=\left(1+|x|^{2}\right)^{-1}\left(1-\Delta_{\xi}\right)
$$

which has the obvious property $L_{\xi} e^{i x \xi}=e^{i x \xi}$. Herewith, and setting $\tilde{u}:=(1-\omega) u$, we obtain the 
required estimate

$$
\begin{aligned}
\left|\partial_{x}^{\alpha} \mathcal{A}_{1}(1-\omega) u\right| & \lesssim \sum_{\alpha_{1}+\alpha_{2}=\alpha}\left|\int \partial_{x}^{\alpha_{1}} a(x, \xi) \xi^{\alpha_{2}} \hat{\tilde{u}}(\xi) e^{i x \xi} d \xi\right| \\
& =\sum_{\alpha_{1}+\alpha_{2}=\alpha}\left|\int \partial_{x}^{\alpha_{1}} a(x, \xi) \xi^{\alpha_{2}} \hat{\tilde{u}}(\xi) L_{\xi}^{m} e^{i x \xi} d \xi\right| \\
& \lesssim\left(1+|x|^{2}\right)^{-m} \sum_{\alpha_{1}+\alpha_{2}=\alpha} \int\left|\left(1-\Delta_{\xi}\right)^{m}\left[\partial_{x}^{\alpha_{1}} a(x, \xi) \xi^{\alpha_{2}} \hat{\tilde{u}}(\xi)\right]\right| d \xi \\
& \lesssim\left(1+|x|^{2}\right)^{-m} \sum_{\alpha_{1}+\alpha_{2}=\alpha} \sum_{\beta_{1}+\beta_{2} \leq 2 m} \int\left|\partial_{\xi}^{\beta_{1}}\left[\partial_{x}^{\alpha_{1}} a(x, \xi) \xi^{\alpha_{2}}\right] \partial_{\xi}^{\beta_{2}} \hat{\tilde{u}}(\xi)\right| d \xi \\
& \lesssim\left(1+|x|^{2}\right)^{-m} \sum_{\beta_{1}+\beta_{2} \leq 2 m}\left\|(1+|\xi|)^{n+|\alpha|-\left|\beta_{1}\right|}\right\|\left\|_{L^{2}}\right\| x^{\beta_{2}} \tilde{u} \|_{L^{2}} \\
& \lesssim\left(1+|x|^{2}\right)^{-m}, \text { for all } m \in \mathbb{Z}_{+} \text {and }|\alpha| \leq s .
\end{aligned}
$$

Remark 3. In order to prove the estimate (2.37) it is sufficient that the symbol of the pseudodifferential operator $\mathcal{A}_{1}$ belongs to $S^{n}\left(\mathbb{R}^{3} \times \mathbb{R}^{3}\right)$, cf. Ref. [11], this corresponds to the class of all symbols $a(x, \xi) \in C^{\infty}\left(\mathbb{R}^{3} \times \mathbb{R}^{3}\right)$ which satisfy the estimates

$$
\left|\partial_{x}^{\alpha} \partial_{\xi}^{\beta} a(x, \xi)\right| \lesssim(1+|\xi|)^{n-|\beta|},
$$

for all $(x, \xi) \in \mathbb{R}^{3} \times \mathbb{R}^{3}$ and $|\alpha|,|\beta| \geq 0$.

Using (2.34), the convolution can be decomposed according to

$$
\frac{1}{|x|} *\left(\tilde{\phi}_{i} \tilde{\phi}_{j}\right)=-\mathcal{P}_{m}\left(\tilde{\phi}_{i} \tilde{\phi}_{j}\right)+(-1)^{m} c^{m} \frac{1}{|x|} * \mathcal{P}_{c}^{m}\left(\tilde{\phi}_{i} \tilde{\phi}_{j}\right)-\frac{1}{|x|} * \mathcal{G}_{m}\left(\tilde{\phi}_{i} \tilde{\phi}_{j}\right) .
$$

For $|\alpha| \leq s, m$ is chosen such that $2 m>s+3 / 2$. According to Proposition 5 and Lemma 2 it is sufficient to consider only the first term in (2.38). The problem can be further reduced by performing a canonical decomposition

$$
\mathcal{P}_{m}=\mathcal{P}_{m}^{\prime}+\tilde{\mathcal{P}}_{m}+\tilde{\mathcal{G}}_{m} \text { with } \mathcal{P}_{m}^{\prime}:=\eta \mathcal{P}_{0} \tilde{\eta} \text { and } \tilde{\mathcal{P}}_{m}:=(1-\eta) \mathcal{P}_{1}(1-\tilde{\tilde{\eta}}),
$$

for appropriately chosen cut-off functions, i.e. $\tilde{\eta} \equiv 1$ on $\operatorname{supp} \eta, \eta \equiv 1$ on $\operatorname{supp} \tilde{\tilde{\eta}}$ and $\omega \tilde{\eta}=\tilde{\eta}$, which yields

$$
\mathcal{P}_{m}\left(\tilde{\phi}_{i} \tilde{\phi}_{j}\right)=\tilde{\mathcal{P}}_{m}\left(\tilde{\phi}_{i} \tilde{\phi}_{j}\right)+\tilde{\mathcal{G}}_{m}\left(\tilde{\phi}_{i} \tilde{\phi}_{j}\right) .
$$

It remains to show how to express $\tilde{\mathcal{P}}_{m}\left(\tilde{\phi}_{i} \tilde{\phi}_{j}\right)$ in such a manner that Proposition 5 can be applied. For this we use again the paramterix of the linear part of the Fock operator i.e. $\mathcal{P}_{i}\left(\mathfrak{h}_{\text {lin }}-\varepsilon_{i}\right)=1+\mathcal{G}_{i}$, which yields the following expression for the eigenfunctions

$$
-\phi_{i}=\mathcal{P}_{i}\left(V_{H} \phi_{i}\right)+\sum_{j} \mathcal{P}_{i}\left(\mathcal{U}_{i, j} \phi_{j}\right)+\mathcal{G}_{i} \phi_{i}
$$

Using a canonical decomposition of the parametrix (2.39) and an appropriately chosen cut-off function $\eta_{1}$, equation (2.40) yields

$$
-\tilde{\phi}_{i}=\tilde{\mathcal{P}}_{i} \tilde{V}_{H} \tilde{\phi}_{i}+\sum_{j} \tilde{\mathcal{P}}_{i} \tilde{\mathcal{U}}_{i, j} \tilde{\phi}_{j}+\tilde{\mathcal{G}}_{i} \phi_{i}, \quad \text { with } \tilde{V}_{H}:=\left(1-\eta_{1}\right) V_{H}, \quad \tilde{\mathcal{U}}_{i, j}:=\left(1-\eta_{1}\right) \mathcal{U}_{i, j}
$$


It is easy to see that the functions $\tilde{V}_{H}$ and $\tilde{\mathcal{U}}_{i, j}$ have bounded derivatives and therefore have been considered here and in the following as multiplicative pseudo-differential operators with $\xi$ independent symbols in $S^{0}\left(\mathbb{R}^{3}\right)$, cf. Remark 3 . The same holds for the functions $\tilde{\phi}_{i}$, with this we get the expression

$$
\tilde{\mathcal{P}}_{m}\left(\tilde{\phi}_{i} \tilde{\phi}_{j}\right) \equiv\left\{\tilde{\mathcal{P}}_{m} \tilde{\phi}_{i}\right\} \tilde{\phi}_{j}=-\left\{\tilde{\mathcal{P}}_{m} \tilde{\phi}_{i} \tilde{\mathcal{P}}_{j} \tilde{V}_{H}\right\} \tilde{\phi}_{j}-\sum_{k}\left\{\tilde{\mathcal{P}}_{m} \tilde{\mathcal{P}}_{j} \tilde{\mathcal{U}}_{j, k}\right\} \tilde{\phi}_{k}-\tilde{\mathcal{P}}_{m}\left(\tilde{\phi}_{i} \tilde{\mathcal{G}}_{j} \tilde{\phi}_{j}\right)
$$

where the terms in curly brackets denote composite operators within the algebra of pseudo-differential operators on $\mathbb{R}^{3}$. We notice that $\left\{\tilde{\mathcal{P}}_{m} \tilde{\phi}_{i} \tilde{\mathcal{P}}_{j} \tilde{V}_{H}\right\}$ and $\left\{\tilde{\mathcal{P}}_{m} \tilde{\mathcal{P}}_{j} \tilde{\mathcal{U}}_{j, k}\right\}$ correspond to pseudo-differential operators with symbols in $S^{-4}\left(\mathbb{R}^{3}\right)$. Furthermore, we remark that $\tilde{\phi}_{i} \tilde{\mathcal{G}}_{j} \tilde{\phi}_{j}$ belongs to $\mathcal{S}\left(\mathbb{R}^{3}\right)$ and that $\tilde{\mathcal{P}}_{m}$ maps $\mathcal{S}\left(\mathbb{R}^{3}\right)$ into $\mathcal{S}\left(\mathbb{R}^{3}\right)$. We can now consecutively insert (2.41) into (2.42) generating pseudo-differential operators of even lower order modulo higher order operators acting on functions from $\mathcal{S}\left(\mathbb{R}^{3}\right)$. This argument enables us to apply the estimate (2.37) and therefore completes the proof that the solutions of the HF equation belong to $\mathcal{S}_{P}^{\gamma}\left(X^{\wedge}\right)$.

\section{Background on the analysis near conical singularities}

This section has the character of an appendix on general tools around elliptic operators near conical singularities or conical exits to infinity.

\subsection{Ellipticity and parametrices near conical singularities}

In general descriptions it makes things more transparent when we admit the operators to be of arbitrary order $m$, not necessarily of order $m=2$, since the parametrices are always of opposite order and the calculus also employs compositions, and, in particular, operators of order zero. Given a differential operator $\tilde{A}$ in $\mathbb{R}^{n+1} \ni x$ with smooth coefficients, polar coordinates $x \rightarrow(r, \alpha)$ in $\mathbb{R}^{n+1} \backslash\{0\},(r, \alpha) \in \mathbb{R}_{+} \times X, X:=S^{n}$, transform $\tilde{A}$ into an operator of the form

$$
A=r^{-m} \sum_{j=0}^{m} a_{j}(r)\left(-r \frac{\partial}{\partial r}\right)^{j},
$$

with coefficients $a_{j}(r) \in C^{\infty}\left(\overline{\mathbb{R}}_{+}, \operatorname{Diff}^{m-j}(X)\right)$; here $\operatorname{Diffl}^{l}(X)$ denotes the space of all differential operators on $X$ of order $l$ with (in local coordinates) smooth coefficients. Operators of the form (3.1) are much more general than operators going back to $\tilde{A}$ with smooth coefficients across the origin in $\mathbb{R}^{n+1}$. In our case we may replace $a_{0}(r)$ by $a_{0}(r)+r^{k}$ for any $k \in \mathbb{N}$, which corresponds in the original operator to an extra potential term $|x|^{-m+k}$ which is singular at $|x|=0$ when $-m+k<0$. This is just the situation in equation (2.2) for $m=2$ and $k=1$ (up to a factor at the potential). One of the main observations on elliptic equations $A u=f$ in $\mathbb{R}_{+} \times X\left(\equiv \mathbb{R}^{n+1} \backslash\{0\}\right)$ is that the solvability depends on chosen weights $\gamma \in \mathbb{R}$ in the spaces $\mathcal{H}^{s, \gamma}\left(X^{\wedge}\right)$ (see the formula (1.9)) and that solutions have asymptotic expansions of the form (1.11) as $r \rightarrow 0$, where $c_{j k} \in L_{j} \subset C^{\infty}(X)$, $p_{j} \in \mathbb{C}, 0 \leq k \leq m_{j}, j \in \mathbb{N}$. Here $\Re p_{j}<\frac{n+1}{2}-\gamma, \Re p_{j} \rightarrow-\infty$ as $j \rightarrow \infty$. Let us briefly explain the general mechanism of how such asymptotics appear and in which way they depend on the individual operator. First of all the ellipticity does not only refer to the homogeneous principal symbol of $A$ of order $m$ which is a smooth function $\sigma_{\psi}(A)(r, \alpha, \varrho, \xi)$ on the cotangent bundle of $X^{\wedge} \ni(r, \alpha)$ minus the zero section $(\varrho, \xi)=0$, but also on a reduced symbol

$$
\tilde{\sigma}_{\psi}(A)(r, \alpha, \varrho, \xi):=r^{m} \sigma_{\psi}(A)\left(r, \alpha, r^{-1} \varrho, \xi\right),
$$

which is smooth up to $r=0$, and in addition on the principal conormal symbol

$$
\sigma_{M}(A)(z):=\sum_{j=0}^{m} a_{j}(0) z^{j} \quad: \quad H^{s}(X) \rightarrow H^{s-m}(X),
$$


which is operator-valued, depending as a holomorphic function on $z \in \mathbb{C}$. It is known that when $\tilde{\sigma}_{\psi}(A) \neq 0$ for $(\varrho, \xi) \neq 0$, up to $r=0$, the operator function (3.2) is a family of Fredholm operators on $X$ of index zero, and there is a countable set $D=D_{A} \subset \mathbb{C}$, where $\left\{c \leq \Re z \leq c^{\prime}\right\} \cap D$ is finite for every $c \leq c^{\prime}$, such that (3.2) consists of isomorphisms for all $z \in \mathbb{C} \backslash D$. Constructing a parametrix of $A$ close to $r=0$ within the so-called cone algebra requires to invert the symbolic components $\left(\sigma_{\psi}(A), \sigma_{M}(A)(z)\right)$. The second component $\sigma_{M}^{-1}(A)(z)$ is a meromorphic operator function with poles at the points $p \in D$ of certain finite multiplicities and Laurent coefficients of the principal part belonging to finite rank operators on $X$ with kernels in $C^{\infty}(X \times X)$. The ellipticity of $A$ with respect to $\sigma_{M}(A)$ and the weight $\gamma \in \mathbb{R}$ is just the condition that the line $\left\{z: \Re z=\frac{n+1}{2}-\gamma\right\}$ does not intersect the set $D$ for $n=\operatorname{dim} X$. A parametrix of $A$ near $r=0$ may be found in form of a Mellin pseudo-differential operator

$$
r^{m} \mathrm{op}_{M}^{\gamma-m-\frac{n}{2}}(h)+M+G,
$$

where $h(r, z)$ is a holomorphic function in $z$, smoothly depending on $r$ up to zero, with values in classical pseudo-differential operators on $X$. The notation means

$$
\mathrm{op}_{M}^{\delta}(f) u(r, x):=r^{\delta} \mathcal{M}^{-1}\left(T^{-\delta} f\right) \mathcal{M} r^{-\delta} u(r, x),
$$

where $\left(T^{-\delta} f\right)(r, z):=f(r, z-\delta)$ for any $\delta \in \mathbb{R}$ and any Mellin symbol $f$ as described before. Furthermore

$$
(\mathcal{M} v)(z)=\int_{0}^{\infty} r^{z-1} v(r) d r \text { and }\left(\mathcal{M}^{-1} g\right)(r)=\frac{1}{2 \pi i} \int_{\Re z=1 / 2} r^{-z} g(z) d z
$$

are the standard Mellin transform and its inverse, respectively. The operator $M$ is a linear combination of expressions of the form

$$
\omega r^{m+j} \operatorname{op}_{M}^{\delta_{j}-m-\frac{n}{2}}\left(f_{j}\right) \tilde{\omega}, j \in \mathbb{N}
$$

with cut-off functions $\omega(r), \tilde{\omega}(r)$, meromorphic Mellin symbols $f_{j}(z)$ with values in smoothing operators on $X$, Schwartz functions in $\Im z$ for $|\Im z| \rightarrow \infty$, and poles, multiplicities and Laurent coefficients of a similar kind as described before in connection with $\sigma_{M}^{-1}(A)(z)$. The weights $\delta_{j}$ are chosen in such a way that $\gamma-m-j<\delta_{j}-m<\gamma-m$ and that the line $\Re z=\frac{n+1}{2}-\left(\delta_{j}-m\right)$ does not intersect the sequence of poles of $f_{j}$. The operator $G$ in (3.3) is of Green type and maps distributions in $\omega \mathcal{H}^{s-m, \gamma-m}\left(X^{\wedge}\right)$ to functions in $\mathcal{H}_{Q, l o c}^{\infty, \gamma}\left(X^{\wedge}\right)$ for some asymptotic type $Q$ (see the notation in Section 1.2; the loc-notation means $\mathcal{H}_{Q}^{\infty, \gamma}\left(X^{\wedge}\right)$ after multiplication by a cut-off function). With it, the inverse of the conormal symbol has the form

$$
\sigma_{M}^{-1}(A)(z)=h(0, z)+f_{0}(z) .
$$

In order to complete the information to a calculus on $X^{\wedge}$ with conical exit to infinity we have to focus on what happens for $r \rightarrow \infty$.

\subsection{Ellipticity and parametrices near a conical exit to infinity}

Our next objective is to consider operators on $\mathbb{R}_{+} \times X$ from the point of view of ellipticity up to $r=\infty$. Since the consideration can be localized with respect to the variable on $X$, the cylinder $\mathbb{R}_{+} \times U$ for a coordinate neighbourhood $U$ on $X$ can be identified with a conical subset of $\mathbb{R}^{n+1}$. The relationship between points on $\mathbb{R}_{+} \times U \ni(r, \alpha)$ and points in $\mathbb{R}^{n+1} \backslash\{0\}$ can be formulated by passing from $U$ via a diffeomorphism to an open subset $V$ in the open unit ball $B \subset \mathbb{R}^{n}$, with $y \in \mathbb{R}^{n}$, such that $\bar{V} \subset B$ and then identifying $(r, \alpha)$ with $(r, r y)$ where $y$ corresponds to $\alpha$ via 
$U \rightarrow V$. Thus setting $x=(r, r y), r>0$, we now assume for convenience that our differential operator $A$, the ellipticity of which at $|x|=\infty$ we are considering, is given globally in $\mathbb{R}^{n+1} \ni x$. Symbols refer to $(x, \xi)$, where $\xi$ is the covariable to $x$ (clearly everything can be rephrased again in polar coordinates). To understand phenomena it is enough to study ellipticity also across $x=0$ which will be cutted out in the final cone calculus on $X^{\wedge}$ and replaced near zero by an operator in the cone calculus. Within this setting, we denote by $S^{m, 0}\left(\mathbb{R}^{n+1} \times \mathbb{R}^{n+1}\right)$ the space of all symbols $a(x, \xi) \in C^{\infty}\left(\mathbb{R}^{n+1} \times \mathbb{R}^{n+1}\right)$ which satisfy the estimates

$$
\left|\partial_{x}^{\alpha} \partial_{\xi}^{\beta} a(x, \xi)\right| \lesssim(1+|\xi|)^{m-|\beta|}(1+|x|)^{-|\alpha|},
$$

for all $(x, \xi) \in \mathbb{R}^{n+1} \times \mathbb{R}^{n+1}$ and $|\alpha|,|\beta| \geq 0$. Different types of principal symbols have to be considered. First, a differential operator

$$
A=\sum_{|\alpha| \leq m} a_{\alpha}(x) D_{x}^{\alpha}
$$

with coefficients $a_{\alpha} \in C^{\infty}\left(\mathbb{R}^{n+1}\right)$ has its standard homogeneous principal symbol

$$
\sigma_{\psi}(A)(x, \xi)=\sum_{|\alpha|=m} a_{\alpha}(x) \xi^{\alpha} \in C^{\infty}\left(\mathbb{R}^{n+1} \times\left(\mathbb{R}^{n+1} \backslash\{0\}\right)\right) .
$$

At $|x|=\infty$ we assume the coefficients $a_{\alpha}$ to be classical symbols in $x$ of order zero. In particular, we can form

$$
\sigma_{e}(A)(x, \xi):=\sum_{|\alpha| \leq m} \sigma_{e}^{0}\left(a_{\alpha}\right)(x) \xi^{\alpha} \in C^{\infty}\left(\left(\mathbb{R}^{n+1} \backslash\{0\}\right) \times \mathbb{R}^{n+1}\right),
$$

where $\sigma_{e}^{0}\left(a_{\alpha}\right)(x)$ denotes the homogeneous principal component of $a_{\alpha}$ of order zero in $x \neq 0$. Finally (3.4) has again a homogeneous principal symbol in $\xi \neq 0$.

$$
\sigma_{\psi, e}(A)(x, \xi):=\sum_{|\alpha|=m} \sigma_{e}^{0}\left(a_{\alpha}\right)(x) \xi^{\alpha} \in C^{\infty}\left(\left(\mathbb{R}^{n+1} \backslash\{0\}\right) \times\left(\mathbb{R}^{n+1} \backslash\{0\}\right)\right) .
$$

The operator $A$ is said to be elliptic globally in $\mathbb{R}^{n+1}$ with respect to the symbolic components

$$
\sigma(A)=\left(\sigma_{\psi}(A), \sigma_{e}(A), \sigma_{\psi, e}(A)\right)
$$

if $\sigma_{\psi}(A)(x, \xi) \neq 0$ for $(x, \xi) \in \mathbb{R}^{n+1} \times\left(\mathbb{R}^{n+1} \backslash\{0\}\right), \sigma_{e}(A)(x, \xi) \neq 0$ for $(x, \xi) \in\left(\mathbb{R}^{n+1} \backslash\{0\}\right) \times \mathbb{R}^{n+1}$, and $\sigma_{\psi, e}(A)(x, \xi) \neq 0$ for $(x, \xi) \in\left(\mathbb{R}^{n+1} \backslash\{0\}\right) \times\left(\mathbb{R}^{n+1} \backslash\{0\}\right)$. Simple examples show that these three conditions are independent. For instance,

$$
A=\Delta-c \text { for } c>0
$$

is elliptic in that sense (we have $m=2$ in this case), while $A=\Delta$ is not. A standard theorem of that calculus tells us that $A$ is elliptic with respect to (3.5) if and only if

$$
A: H^{s}\left(\mathbb{R}^{n+1}\right) \rightarrow H^{s-m}\left(\mathbb{R}^{n+1}\right)
$$

is a Fredholm operator for any fixed $s=s_{0} \in \mathbb{R}$ (this entails then the Fredholm property for all $s \in \mathbb{R}$ ). If $A$ is elliptic, then there is a parametrix $P$ which is a classical pseudo-differential operator in $\mathbb{R}^{n+1}$, again with a triple of symbols $\sigma(P)=\left(\sigma_{\psi}(P), \sigma_{e}(P), \sigma_{\psi, e}(P)\right)$, the components of which are inverse to the ones in (3.5). The operator $P$ satisfies the relation

$$
P A=1+C_{l}, \quad A P=1+C_{r},
$$

with smoothing operators $C_{l}$ and $C_{r}$ which are integral operators with kernels in $\mathcal{S}\left(\mathbb{R}^{n+1} \times \mathbb{R}^{n+1}\right)$. 


\subsection{The cone calculus}

By cone calculus, here on $X^{\wedge} \ni(r, \alpha)$ with $r \rightarrow 0$ coresponding to a conical singularity, $r \rightarrow \infty$ to a conical exit, we understand a calculus of operators

$$
A=r^{-m} \omega \mathrm{op}^{\gamma-\frac{n}{2}}(h) \tilde{\omega}+(1-\omega) A_{\text {int }}(1-\tilde{\tilde{\omega}})+M+G,
$$

where $\omega, \tilde{\omega}, \tilde{\tilde{\omega}}$ are cut-off functions on the half-axis, $\tilde{\omega} \equiv 1$ on $\operatorname{supp} \omega, \omega \equiv 1$ on $\operatorname{supp} \tilde{\tilde{\omega}}$, such that $h(r, z)$ is $C^{\infty}$ in $r \in \overline{\mathbb{R}}_{+}$, holomorphic in $z \in \mathbb{C}$, such that $h(r, \beta+i \varrho)$ is a parameter-dependent pseudo-differential operator of order $m \in \mathbb{R}$ on $X$ with parameter $\varrho, A_{\text {int }}$ is a pseudo-differential operator of order $m$ on $\mathbb{R}^{n+1}$ with exit symbolic structure as explained in the preceding section, $M$ is as in Section 3.1, while $G$ is a Green operator on $X^{\wedge}$ with discrete asymptotics for $r \rightarrow 0$, that means

$$
G: \mathcal{K}^{s, \gamma}\left(X^{\wedge}\right) \rightarrow \mathcal{S}_{Q}^{\gamma-m}\left(X^{\wedge}\right)
$$

is continuous for every $s \in \mathbb{R}$, with some asymptotic type $Q$, and the formal adjoint with respect to the $\mathcal{K}^{0,0}\left(X^{\wedge}\right)$-scalar product has a similar property. We denote by $C^{m}\left(X^{\wedge}, \underline{g}\right)$, the class of all operators (3.6) which can be associated to specific weight data $\underline{g}$.

The operators (3.6) belong to the space of classical pseudo-differential operators of order $m$ on the open $C^{\infty}$ manifold $\mathbb{R}_{+} \times X$ with $\sigma_{\psi}(A)$, the standard homogenous principal symbol of order $m$. In this connexion ellipticity also refers to the reduced symbol $\tilde{\sigma}_{\psi}(A)$ near $r=0$, moreover, to the conormal symbol $\sigma_{M}(A)(z), \Re z=\frac{n+1}{2}-\gamma$ for a weight $\gamma \in \mathbb{R}$, and to the exit symbolic components $\sigma_{e}(A)$ and $\sigma_{\psi, e}(A)$. This has been explained in the preceding sections. Observe that every operator (3.6) induces continuous operators

$$
A: \mathcal{K}^{s, \gamma}\left(X^{\wedge}\right) \rightarrow \mathcal{K}^{s-m, \gamma-m}\left(X^{\wedge}\right)
$$

and

$$
\begin{gathered}
A: \mathcal{K}_{Q}^{s, \gamma}\left(X^{\wedge}\right) \rightarrow \mathcal{K}_{R}^{s-m, \gamma-m}\left(X^{\wedge}\right), \\
A: \mathcal{S}_{Q}^{\gamma}\left(X^{\wedge}\right) \rightarrow \mathcal{S}_{R}^{\gamma-m}\left(X^{\wedge}\right),
\end{gathered}
$$

for every $s \in \mathbb{R}$ and every asymptotic type $Q$ with some resulting asymptotic type $R$ (which also depends on $A$, more precisely, on the meromorphic structure of the involved Mellin symbols).

Theorem 3. For an operator (3.6) the following conditions are equivalent:

(i) A is elliptic ( with respect to $\gamma$ what concerns $\sigma_{M}(A)$ ).

(ii) A induces a Fredholm operator (3.8).

Theorem 4. An elliptic operator (3.6) has a parametrix $P$ of analogous structure, of order $-m$, elliptic with respect to the weight $\gamma-m$, which means

$$
P A=1+G_{l}, \quad A P=1+G_{r},
$$

with some Green operators $G_{l}, G_{r}$. Moreover, $A u=f \in \mathcal{K}_{(R)}^{s-m, \gamma-m}\left(X^{\wedge}\right)$ and $u \in \mathcal{K}^{-\infty, \gamma}\left(X^{\wedge}\right)$ entails $u \in \mathcal{K}_{(Q)}^{s, \gamma}\left(X^{\wedge}\right)$ for every $s \in \mathbb{R}$ and every asymptotic type $R$ with a resulting asymptotic type $Q$ ( parentheses mean that such a result is valid also with respect to the spaces without control of asymptotics ).

Corollary 2. $A u=0$ entails $u \in \mathcal{S}_{Q}^{\gamma}\left(X^{\wedge}\right)$ for some asymptotic type $Q$.

Proof. Choose a parametrix $P$ of $A$ and compute $P A=1+G_{l}$. Then $A u=0$ implies $\left(1+G_{l}\right) u=0$; however, the relation (3.7) gives us $u \in \mathcal{S}_{Q}^{\gamma}\left(X^{\wedge}\right)$.

\section{Acknowledgements}

This work was supported by the Deutsche Forschungsgemeinschaft (SPP 1145). 


\section{References}

[1] E. Cancès, SCF algorithms for HF electronic calculations, in M. Defranceschi, and C. Le Bris (Eds.), Mathematical Models and Methods for Ab Initio Quantum Chemistry, Lecture Notes in Chemistry 74, 17-43 (Springer, Berlin, 2000).

[2] E. Cancès, and C. Le Bris, On the convergence of SCF algorithms for the Hartree-Fock equations, ESAIM: M2AN 34, 749-774 (2000).

[3] Y. V. Egorov and B.-W. Schulze, Pseudo-Differential Operators, Singularities, Applications (Birkhäuser, Basel, 1997).

[4] H.-J. Flad, W. Hackbusch, and R. Schneider, Best $N$-term approximation in electronic structure calculations. I. One-electron reduced density matrix, ESAIM: M2AN 40, 49-61 (2006).

[5] D. Gilbarg, and N. S. Trudinger, Elliptic Partial Differential Equations of Second Order (Springer, Berlin, 1998).

[6] T. Helgaker, P. Jørgensen, and J. Olsen, Molecular Electronic-Structure Theory (Wiley, New York, 1999).

[7] R. N. Hill, Rates of convergence and error estimation formulas for the Rayleigh-Ritz variational method, J. Chem. Phys. 83, 1173-1196 (1985).

[8] E. H. Lieb, and B. Simon, The Hartree-Fock theory for Coulomb systems, Commun. Math. Phys. 53, 185-194 (1977).

[9] P. L. Lions, Solutions of Hartree-Fock equations for Coulomb systems, Commun. Math. Phys. 109, 33-97 (1987).

[10] B.-W. Schulze, Boundary Value Problems and Singular Pseudo-Differential Operators (Wiley, New York, 1998).

[11] E. M. Stein, Harmonic Analysis: Real-Variable Methods, Orthogonality, and Oscillatory Integrals (Princeton University Press, Princeton, 1993).

[12] H. Triebel, Higher Analysis (Barth, Leipzig, 1992). 